\title{
Induced modules for vertex operator algebras
}

\author{
Chongying Dong and Zongzhu Lin
}

\begin{abstract}
For a vertex operator algebra $V$ and a vertex operator subalgebra $V^{\prime}$ which is invariant under an automorphism $g$ of $V$ of finite order, we introduce a $g$-twisted induction functor from the category of $g$-twisted $V^{\prime}$-modules to the category of $g$-twisted $V$-modules. This functor satisfies the Frobenius reciprocity and transitivity. The results are illustrated with $V^{\prime}$ being the $g$-invariants in simple $V$ or $V^{\prime}$ being $g$-rational.
\end{abstract}

\section{Introduction}

A lot of progress on the representation theory for vertex operator algebras has been made in the last few years. For example, the representation theory for the concrete vertex operator algebras, which include the moonshine vertex operator algebra $V^{\natural}$ ([FML],[D3]), the vertex operator algebras based on even positive definite lattices [D1], the vertex operator algebras associated with the integrable representations of affine Lie algebras and Virasoro algebras ([DMZ], [DL], [FZ], [W]), have been studied extensively. There are also abstract approaches such as Zhu's one to one correspondence between the set of inequivalent irreducible modules for a given vertex operator algebra and the set of inequivalent irreducible modules for an associative algebra associated with the vertex operator algebra [Z], and the tensor products of modules ([HL] and [L]); See also [FHL] for the results concerning intertwining operators and contragredient modules. Many of these results are analogues of the corresponding results in the classical Lie algebra theory.

The purpose of this paper is to give a construction of induced twisted modules for vertex operator algebras and present some initial results. The main idea in constructing the induced module comes from the induction theory for the representations of Lie groups, algebraic groups, quantum groups, Hopf algebras ([V], [J], [APW], [Lin1-Lin2]). In order for the induced module to have the functorial property, one has to enlarge the

1991 Mathematics Subject Classification Primary 17B35

The first author was supported by NSF grant DMS-9303374 and a research grant from the Committee on Research, UC Santa Cruz.

The second author was supported by NSF grant MDS-9401389. 
category of $g$-twisted modules to ensure the existence of the induced modules. This resembles Harish-Chandra's theory in the representation theory of Lie groups. We prove that in most interesting cases, the induced modules from a simple module for a vertex operator subalgebra are indeed $g$-twisted modules. The structures of these modules in certain special cases are discussed.

One of the main motivations for introducing the induced modules is to study the "orbifold conformal field theory." Roughly speaking, an orbifold theory is a conformal field theory which is obtained from a given conformal field theory modulo the action of a finite symmetry group (see [DVVV]). Let $V$ be a vertex operator algebra and $G$ be a finite subgroup of the automorphisms of $V$. Denote by $V^{G}$ the subspace of $V$ consisting of the fixed points under the action of $G$. Then $V^{G}$ is a vertex operator subalgebra of $V$. Algebraically, the orbifold theory is to study the representation theory of $V^{G}$. The main new feature of the orbifold theory is the introduction of twisted modules. A $g$-twisted module for $g \in G$ is automatically an ordinary module for $V^{G}$ under the restriction. It is proved in [DM2] that if $V$ is holomorphic and $G$ is nilpotent then any irreducible $g$-twisted $V$-module is completely reducible as a $V^{G}$-module. It is conjectured that this is true for arbitrary $G$ and that any irreducible $V^{G}$-module appears as an irreducible component of some irreducible twisted module (see e.g. [DVVV], [DPR] and [DM2]). The theory of induced twisted modules for vertex operator algebras, discussed in this paper, is being developed with this conjecture in mind.

This paper is organized as follows: In Section 2, after recalling the notion of twisted module for a vertex operator algebra from [D2] and [FFR], we define the twisted enveloping algebra $A(g)$. We give a necessary and sufficient condition under which an $A(V)$-module is a $g$-twisted module. A linear topology is defined on $A(g)$ by a topological basis at 0 consisting of all $\operatorname{Ann}_{A(g)}(m)$ for an element $m$ in a twisted module. It turns out this topology is the weakest topology on $A(g)$ so that the $A(g)$-module structure on any twisted module $M$ gives a continuous map from $A(g)$ to $\operatorname{End}_{\mathbb{C}}(M)$, which is equipped with the point-wise convergence topology. It is important to note that we need the representations to be continuous in order for the Jacobi identity to hold. We also introduce a certain $A(g)$-module category $\overline{\mathcal{C}}_{g}$ which contains the $g$ twisted $V$-module category $\mathcal{C}_{g}$ as a full subcategory. In fact, $\overline{\mathcal{C}}_{g}$ consists of the objects of $\mathcal{C}_{g}$ together with their direct limits in the category of $A(g)$-modules.

Section 3 which devotes the definition of induced module is the center of the paper. Let $g$ be a finite order automorphism of $V$ and $V^{\prime}$ a subalgebra of $V$ which is $g$-invariant. Denote the restriction of $g$ to $V^{\prime}$ by $g^{\prime}$. Then there is an algebra embedding from $A\left(g^{\prime}\right)$ into $A(g)$. For a $g^{\prime}$-twisted $V^{\prime}$-module $W$ we define $\operatorname{Ind}_{\overline{\mathcal{C}}_{g^{\prime}}^{\prime}}^{\overline{\mathcal{C}}_{g}}(W)$ to be the subspace of $\operatorname{Hom}_{A\left(g^{\prime}\right)}(A(g), W)$ consisting of elements which are killed by some $\operatorname{Ann}_{A(g)}(m)$ for some $m$ in a $g$-twisted $V$-module. Then we prove that this induction functor enjoys all the properties of an induction functor should have, such as the Frobenius reciprocity and the transitivity.

In Section 4 we investigate the $g$-induced modules for vertex operator algebra with 
only finitely many irreducible $g$-twisted modules. In the case that $V$ is $g$-rational, that is, any $g$-twisted module is completely reducible, we show that the induced module of $W$, which has a composition series, from a $g$-invariant subalgebra to $V$ is in fact in $\mathcal{C}_{g}$. In particular, the induced module from any irreducible module in this case is an ordinary $g$-twisted module.

In Section 5 we discuss the induction from a subalgebra which is the fixed points of an finite automorphism. First we show that the cyclic group $\langle g\rangle$ acts on any irreducible $g$-twisted module, each eigenspace of $g$ is an irreducible $V^{G}$-module, different eigenspaces are inequivalent $V^{G}$-modules, and the eigenspaces from inequivalent irreducible $g$-twisted modules are inequivalent $V^{G}$-modules. Using this result together with Frobenius reciprocity we show that the $g$-induced module of an eigenspace of $g$ in an irreducible $g$-twisted module $W$ from $V^{G}$ to $V$ has a unique simple $g$-twisted submodule isomorphic to $W$, and the $g$-induced modules from any other irreducible $V^{G}$ module is zero.

\section{Twisted enveloping algebras and their modules.}

2.1. Twisted modules. Let $(V, Y, \mathbf{1}, \omega)$ be a vertex operator algebra (we refer to $[\mathrm{B}],[\mathrm{FHL}]$ and $[\mathrm{FLM}]$ for definitions and properties) and $g$ be an automorphism of $V$ of finite order $K$. Then $V$ is a direct sum of eigenspaces of $g$ :

$$
V=\oplus_{r \in \mathbb{Z} / K \mathbb{Z}} V^{r}
$$

where $V^{r}=\left\{v \in V \mid g v=e^{r 2 \pi i / K} v\right\}$. (We will use $r \in\{0,1, \ldots, K-1\}$ to denote both the representing residue class and the integer itself.) Following [D2] and [FFR], a $g$-twisted module $M$ for $V$ is a $\mathbb{C}$-graded vector space:

$$
M=\coprod_{\lambda \in \mathbb{C}} M_{\lambda}
$$

(for $w \in M_{\lambda}, \lambda=$ wt $w$ is called the weight of $w$ ) such that for each $\lambda \in \mathbb{C}$

$$
\operatorname{dim} M_{\lambda}<\infty \text { and } M_{n+\lambda}=0 \text { for } n \in \mathbb{Z} \text { sufficiently small; }
$$

and equipped with a linear map

$$
\begin{aligned}
& V \rightarrow(\text { End } M)\{z\} \\
& v \mapsto Y_{M}(v, z)=\sum_{n \in \mathbb{Q}} v_{n} z^{-n-1} \quad\left(v_{n} \in \text { End } M\right)
\end{aligned}
$$

(where for any vector space $W$, we define $W\{z\}$ to be the vector space of $W$-valued formal series in $z$, with arbitrary real powers of $z$ ) satisfying the following conditions 
for $u, v \in V, w \in M$, and $r \in \mathbb{Z} / K \mathbb{Z}$ :

$$
\begin{gathered}
Y_{M}(v, z)=\sum_{n \in \frac{r}{K}+\mathbb{Z}} v_{n} z^{-n-1} \quad \text { for } \quad v \in V^{r} ; \\
v_{l} w=0 \text { for } \quad l \in \mathbb{Q} \text { sufficiently large; } \\
Y_{M}(\mathbf{1}, z)=1 ; \\
z_{0}^{-1} \delta\left(\frac{z_{1}-z_{2}}{z_{0}}\right) Y_{M}\left(u, z_{1}\right) Y_{M}\left(v, z_{2}\right)-z_{0}^{-1} \delta\left(\frac{z_{2}-z_{1}}{-z_{0}}\right) Y_{M}\left(v, z_{2}\right) Y_{M}\left(u, z_{1}\right) \\
=z_{2}^{-1}\left(\frac{z_{1}-z_{0}}{z_{2}}\right)^{-r / K} \delta\left(\frac{z_{1}-z_{0}}{z_{2}}\right) Y_{M}\left(Y\left(u, z_{0}\right) v, z_{2}\right)
\end{gathered}
$$

if $u \in V^{r}$;

$$
[L(m), L(n)]=(m-n) L(m+n)+\frac{1}{12}\left(m^{3}-m\right) \delta_{m+n, 0}(\operatorname{rank} V)
$$

for $m, n \in \mathbb{Z}$, where

$$
\begin{aligned}
& L(n)=\omega_{n+1} \quad \text { for } n \in \mathbb{Z}, \quad \text { i.e., } \quad Y_{M}(\omega, z)=\sum_{n \in \mathbb{Z}} L(n) z^{-n-2} ; \\
& L(0) w=n w=(\text { wt } w) w \text { for } \quad w \in M_{n}(n \in \mathbb{C}) ; \\
& \frac{d}{d z} Y_{M}(v, z)=Y_{M}(L(-1) v, z) .
\end{aligned}
$$

This completes the definition. We denote this module by $\left(M, Y_{M}\right)$ (or briefly by $M$ ).

The following are consequences of the definition

$$
\begin{aligned}
& {\left[L(-1), Y_{M}(v, z)\right]=Y_{M}(L(-1) v, z)} \\
& {\left[L(0), Y_{M}(v, z)\right]=Y_{M}(L(0) v, z)+z Y_{M}(L(-1) v, z) .}
\end{aligned}
$$

Remark. From (5), (6), (7) and (9) we find that if $v \in V$ is homogeneous, then $v_{n}$ has weight wt $v-n-1$ as an operator, that is, $v_{n}: M_{\lambda} \rightarrow M_{\lambda+\mathrm{wt}-\mathrm{n}-1}$. In particular, $L(n)$ has weight $-n$.

Remark. For each $v \in V$, and $u \in V^{r}$ and any $p \in \mathbb{Z}$ and $s, t \in \mathbb{R}$, we can compare the coefficients of $z_{0}^{-p-1} z_{1}^{-s-1} z_{2}^{-t-1}$ on the both sides of the Jacobi identity (5) to get

$$
\sum_{m \geq 0}(-1)^{m}\left(\begin{array}{c}
p \\
m
\end{array}\right)\left(u_{p+s-m} v_{t+m}-(-1)^{p} v_{p+t-m} u_{s+m}\right)=\sum_{m \geq 0}\left(\begin{array}{c}
s \\
m
\end{array}\right)\left(u_{p+m} v\right)_{s+t-m} .
$$

On the other hand, all these identities together will imply the Jacobi identity (5). We will use these identities more often.

A vertex operator algebra $V$ is called $g$-quasi-rational if $V$ has only finitely many irreducible $g$-twisted modules. If $V$ further satisfies the condition that any $g$-twisted module is completely reducible, we call $V$ a $g$-rational vertex operator algebra. If $g=1$, we get a rational vertex operator algebra. 
2.2. Let $M$ and $N$ be two $g$-twisted $V$-modules. A homomorphism $\phi: M \rightarrow N$ of $g$ twisted $V$-modules is a linear map which commutes with the operators $v_{n}$ for all $v \in V$ and $n \in \frac{1}{K} \mathbb{Z}$. In particular, a homomorphism is always homogeneous homomorphism of graded vector spaces in the sense that $\phi\left(M_{\lambda}\right) \subseteq N_{\lambda}$ since $\phi$ commutes with the operator $L(0)$.

Let $\mathcal{C}_{g}(V)$ be the category of $g$-twisted $V$-modules. Then $\mathcal{C}_{g}(V)$ is an abelian category. For simplicity, we will use $\mathcal{C}_{g}$ if the vertex operator algebra $V$ is understood. We will abuse the notation a little by using $M \in \mathcal{C}_{g}$ to stand for $M$ being an object of the category $\mathcal{C}_{g}$.

Let $V^{\prime}$ be another vertex operator algebra and $\phi: V^{\prime} \rightarrow V$ a homomorphism of vertex operator algebras. If $g^{\prime}$ is an automorphism of $V^{\prime}$ such that $\phi \circ g^{\prime}=g \circ \phi$, then $\phi$ sends the $g^{\prime}$-eigenspaces to $g$-eigenspaces. In fact, let $K^{\prime}$ be the order of $g^{\prime}$. If $v^{\prime} \in\left(V^{\prime}\right)^{r^{\prime}}$, then $\phi\left(v^{\prime}\right) \in V^{r}$. Here we have $\frac{r}{K}=\frac{r^{\prime}}{K^{\prime}}$. By examining Jacobi identity, one can verify that every $g$-twisted $V$-module is automatically a $g^{\prime}$-twisted $V^{\prime}$-module. In this way, $\phi$ induces a functor $\mathcal{C}_{g} \rightarrow \mathcal{C}_{g^{\prime}}$, which is exact.

In the sequel, we will describe the category $\mathcal{C}_{g}$ as a full subcategory of the category of all modules for an associated algebra.

2.3. $g$-twisted enveloping algebras. Consider the $\mathbb{C}$-vector space $\bar{V}=\oplus_{i=0}^{K-1}\left(V^{i} \otimes\right.$ $\left.t^{i / K} \mathbb{C}\left[t, t^{-1}\right]\right)$ and the tensor algebra $T(\bar{V})$. Note that $\bar{V}$ is a graded vector space with the gradation $\bar{V}=\oplus_{\mu \in \frac{1}{K} \mathbb{Z}}(\bar{V})_{\mu}$. Here $(\bar{V})_{\mu}=\oplus_{n \in \mathbb{Z}}\left(V_{n} \otimes t^{n-\mu-1}\right)$. Thus the tensor algebra $T(\bar{V})$ is a graded algebra with the gradation induced by the gradation on the vector space $\bar{V}$.

For each $g$-twisted $V$-module $M$, the module structure of $V$ on $M$ induces a linear map

$$
\begin{array}{ccc}
\bar{V} & \rightarrow & \operatorname{End}_{\mathbb{C}}(M) \\
v \otimes t^{n} & \mapsto & v_{n},
\end{array}
$$

where $v_{n}$ is the component operator of $Y_{M}(v, z)=\sum_{n \in \frac{1}{K} \mathbb{Z}} v_{n} z^{-n-1}$, which extends uniquely to a homomorphism of associative algebras

$$
\rho_{M}: T(\bar{V}) \rightarrow \operatorname{End}(M) .
$$

Let $\operatorname{End}_{\mathbb{C}}(M)_{\mu}=\left\{f \in \operatorname{End}_{\mathbb{C}}(M) \quad \mid f\left(M_{\lambda}\right) \subseteq M_{\lambda+\mu}\right.$ for all $\left.\lambda \in \mathbb{C}\right\}$. Then the $\mathbb{C}$-graded algebra Endgr $(M)=\oplus_{\mu \in \mathbb{C}} \operatorname{End}_{\mathbb{C}}(M)_{\mu}$ is a subalgebra of $\operatorname{End}_{\mathbb{C}}(M)$. By the definition of the homomorphism $\rho_{M}$, one can see that $\rho_{M}(T(\bar{V})) \subseteq \operatorname{Endgr}(M)$ and the map $\rho_{M}: T(\bar{V}) \rightarrow \operatorname{Endgr}(M)$ is a homomorphism of graded algebras. Thus the kernel $\operatorname{Ker}\left(\rho_{M}\right)$ is a graded ideal of $T(\bar{V})$.

Set $I=\cap_{M \in \mathcal{C}_{g}} \operatorname{ker} \rho_{M}$. Then $I$ is also a graded ideal of $T(\bar{V})$. We define $U_{g}(V)=$ $T(\bar{V}) / I$, which is also a $\frac{1}{K} \mathbb{Z}$-graded algebra with

$$
U_{g}(V)=\oplus_{n \in \frac{1}{K} \mathbb{Z}}\left(U_{g}(V)\right)_{n}
$$


such that

$$
\mathrm{wt}\left(v \otimes t^{n}\right)=\mathrm{wt} v_{n}=\mathrm{wt} v-n-1
$$

for homogeneous $v \in V$. We will also use $v_{n}$ to denote the image of $v \otimes t^{n}$ in $U_{g}(V)$ acting on any $g$-twisted $V$-modules. Consider the Lie algebra $s l_{2}$ generated by the operators $\{L(1), L(0), L(-1)\}$. Then $U_{g}(V)$ becomes an $s l_{2}$-module with $L(1), L(0)$, and $L(-1)$ acting on $U_{g}(V)$ as derivations. As $s l_{2}$-module, $U_{g}(V)$ is a direct sum of $L(0)$-weight spaces, which gives $U_{g}(V)$ the graded algebra structure. Furthermore, any $g$-twisted modules are automatically modules for $s l_{2}$ with weight space decomposition defining the graded vector space structure.

Lemma (1) Every $g$-twisted $V$-module $M$ is naturally a graded left $U_{g}(V)$-module with the graded structure given by the weight spaces decomposition as $s l_{2}$-module.

(2) Any $U_{g}(V)$-submodules and quotient $U_{g}(V)$-modules of a $g$-twisted $V$-module $M$ are also $g$-twisted $V$-modules.

(3) For any two $g$-twisted $V$-modules $M, N$, a linear map $\phi: N \rightarrow M$ is a homomorphism of $g$-twisted module if and only if $\phi$ is a homomorphism of $U_{g}(V)$-modules.

Proof: (1) It follows from the construction of $U_{g}(V)$. To prove (2), let $N \subseteq M$ be a $U_{g}(V)$-submodule of $M$. We first show that $N$ is a graded $U_{g}(V)$-submodule of $M$. Let $x \in N$ and $x=\sum x_{\lambda}$ be a finite sum in $M$ with $x_{\lambda} \in M_{\lambda}$ for distinct $\lambda$ 's. We must show that $x_{\lambda} \in N$. We use induction on the number of the nonzero terms of the summation. By applying the operator $L(0)$ (the image of $\omega \otimes t^{1}$ in $U_{g}(V)$ ) we get $\left(L(0)-\lambda_{0}\right) x=\sum\left(\lambda-\lambda_{0}\right) x_{\lambda}$ with a shorter expression. By induction hypothesis, we have $x_{\lambda} \in N$. Now the conditions on the gradation of a $g$-twisted $V$-module is automatically satisfied. If $Y_{M}(v, z)=\sum_{n \in \frac{1}{K} \mathbb{Z}} v_{n} z^{-n-1}$, with $v_{n} \in \operatorname{End}(M)$, we have $v_{n} N \subseteq N$. So we can consider $\left.v_{n}\right|_{N} \in \operatorname{End}(N)$ and define $Y_{N}(v, z)=\left.\sum_{n \in \frac{1}{K} \mathbb{Z}} v_{n}\right|_{N} z^{-n-1}$. Now all other conditions for a $g$-twisted $V$-module structure on $N$ can be verified straightforward. Note that the both sides of the $g$-twisted Jacobi identities make sense as operators on $N$ at every element of $N$. For the quotient, since the kernel is a $U_{g}(V)$-submodule, it is a $g$-twisted $V$-submodule of $M$. Thus the quotient is a graded module and one can verify that the $U_{g}(V)$-structure on the quotient $g$-twisted $V$-module $M / N$ is the same as the quotient of the $U_{g}(V)$-modules.

(3) It is straightforward to verify that any homomorphism of $g$-twisted $V$-modules is naturally a homomorphism of $U_{g}(V)$-modules. Conversely, if a linear map $\phi$ is a $U_{g}(V)$-homomorphism, then it has to commute with the operator $L(0)$. This shows that $\phi\left(N_{\lambda}\right) \subseteq M_{\lambda}$. Since $\phi$ commutes with all $v \otimes t^{n}$, then we have $\phi \circ Y_{N}(v, z)=Y_{M}(v, z) \circ \phi$.

2.4. The Algebra $A(g)$. Note that many of the identities such as the Jacobi identities will involve infinite sum, which does not make any sense in the purely algebraic setting in $U_{g}(V)$. 
For each $\mathbb{C}$-vector space $A$, let $A[[X]]$ be the vector space of formal power series. The natural embedding $A \rightarrow A[[X]]$ (sending elements of $A$ to power series with constant term only) makes $A$ a subspace of $A[[X]]$. If $A$ is further an algebra, then $A[[X]]$ is an algebra containing $A$ as a subalgebra. Here the multiplication is given by the multiplication of formal power series.

Let $M$ be a vector space, and $A=\operatorname{End}(M)$. Then $A[[X]]$ has a subspace

$$
C_{M}=\left\{f(X)=\sum_{i=0}^{\infty} a_{i} X^{i} \in A[[X]] \mid \sum_{i=0}^{\infty} a_{i} m X^{i} \in M[X] \text { for each } m \in M\right\} .
$$

In fact $C_{M}$ is a subalgebra of $A[[X]]$. In this way, we can make $M$ a $C_{M}$-module by defining the action $f(X) m=\sum_{n=0}^{\infty} a_{n} m$.

Consider the vector space $U_{g}(V)_{n}[[X]]$ and the algebra $\oplus_{n \in \frac{1}{K} \mathbb{Z}}\left(U_{g}(V)_{n}[[X]]\right)$, which is a proper subalgebra of $U_{g}(V)[[X]]$. For each $g$-twisted $V$-module $M$, the homomorphism $\rho_{M}: U_{g}(V) \rightarrow \operatorname{End}(M)$ induces a homomorphism $\rho_{M}[X]: \oplus_{n \in \frac{1}{K} \mathbb{Z}} U_{g}(V)_{n}[[X]] \rightarrow$ $\operatorname{End}(M)[[X]]$.

Let $C=\cap_{M \in \mathcal{C}_{g}}\left(\rho_{M}[X]\right)^{-1}\left(C_{\operatorname{End}(M)}\right)$. Then $C$ is a subalgebra of $\oplus_{n \in \frac{1}{K} \mathbb{Z}} U_{g}(V)_{n}[[X]]$. Note that $\oplus_{n \in \frac{1}{K} \mathbb{Z}} U_{g}(V)_{n}[[X]]$ is a $\frac{1}{K} \mathbb{Z}$-graded algebra by defining wt $X=0$ and wt $f h=$ wt $f+$ wth for $f, h \in \oplus_{n \in \frac{1}{K} \mathbb{Z}} U_{g}(V)_{n}[[X]]$. Then $C=\oplus_{n \in \frac{1}{K} \mathbb{Z}} C_{n}$ is a graded subalgebra with $C_{n}=U_{g}(V)_{n}[[X]] \cap C$.

Since $M$ in $\mathcal{C}_{g}$ is a natural graded $C$-module, $\operatorname{Ann}_{C}(M)$ is an graded (two-sided) ideal of $C$. Define $A(g)=C /\left(\cap_{M \in \mathcal{C}_{g}} \operatorname{Ann}_{C}(M)\right)$. Then $A(g)$ is a graded algebra. The constant subalgebra $U_{g}(V)$ of $\oplus_{n \in \frac{1}{K} \mathbb{Z}} U_{g}(V)_{n}[[X]]$ is actually contained in $C$. Moreover we have a natural algebra embedding $U_{g}(V) \rightarrow A(g)$. Note that the $s l_{2}$-module structure on $U_{g}(V)$ extends naturally to $C_{M}$, which has a weight spaces decomposition and the map $\rho_{M}: C \rightarrow \operatorname{End}(M)$ is a homomorphism of $s l_{2}$-modules. Thus the kernels $\operatorname{Ann}_{C}(M)$ are graded ideals. This naturally induces a graded algebra structure on $A(g)$.

2.5. By our construction of the algebra $A(g)$, every $g$-twisted $V$-module extends to an $A(g)$-module. We can now regard $U_{g}(V)$ as a subalgebra of $A(g)$. Thus, every $g$-twisted $V$-module $M$ is a $A(g)$-module and every homomorphism between any two $g$-twisted $V$-modules are also a homomorphism of $A(g)$-modules. This yields a functor $\mathcal{E}: \mathcal{C}_{g} \rightarrow A(g)$-Mod (the category of all $A(g)$-modules).

Theorem The functor $\mathcal{E}$ is an isomorphism of $\mathcal{C}_{g}$ to a full subcategory of $A(g)$-Mod.

Proof: First we show that $\mathcal{E}$ is full. Let $M$ and $N$ be two modules in $\mathcal{C}_{g}$. Then a linear map $f: M \rightarrow N$ is a homomorphism of $g$-twisted $V$-modules if and only if it $U_{g}(V)$ linear and further, if and only if it is $A(g)$-linear. This shows that $\operatorname{Hom}_{\mathcal{C}_{g}}(M, N)=$ 
$\operatorname{Hom}_{A(g)-\operatorname{Mod}}(M, N)$ as vector spaces. This shows that $\mathcal{E}$ is full. The faithfulness and the exactness follow from the fact that $\mathcal{E}$ does not change the vector space.

Let $\mathcal{C}_{A(g)}$ be the full subcategory of $A(g)$-modules satisfying the conditions of (i). Then (i) defines the functor $\mathcal{E}^{-1}$ and this shows that $\mathcal{E}$ is an isomorphism between $\mathcal{C}_{g}$ and $\mathcal{C}_{A(g)}$. This proves the theorem.

From now on, we can simply identify $\mathcal{C}_{g}$ with $\mathcal{C}_{A(g)}$ and still use $\mathcal{C}_{g}$ to denote it.

2.6. Topology on $A(g)$. For each vector space $M, \operatorname{End}_{\mathbb{C}}(M)$ is equipped with a natural product topology (point-wise topology), which is induced from the product topology on $M^{M}$ where $M$ is equipped with the discrete topology.

In particular, if $M$ is a graded vector space, each $\operatorname{End}_{\mathbb{C}}(M)_{\mu}$ is a closed vector subspace of $\operatorname{End}_{\mathbb{C}}(M)$, although $\operatorname{End}_{g r} M$ is not closed in general. A graded vector subspace $E=\oplus_{\mu} E_{\mu}$ of $\operatorname{End}_{\mathbb{C}}(M)$ is called graded closed in $\operatorname{End}_{\mathbb{C}}(M)$ if each $E_{\mu}$ is closed.

We define the topology on $A(g)$ to be the weakest topology such that all induced maps $\rho_{M}: A(g) \rightarrow \operatorname{End}_{\mathbb{C}}(M)$ for $M \in \mathcal{C}_{g}$ are continuous.

For each $m \in M_{\mu}$, the left ideal $\operatorname{Ann}_{A(g)}(m)=\{a \in A(g) \mid a m=0\}$ of $A(g)$ is a graded left ideal of $A(g)$. In fact, if $a \in A(g)$ such that $a=\oplus a_{\lambda}$ (a finite sum with $a_{\lambda} \in A(g)_{\lambda}$ and distinct $\lambda$ 's), then $a m=0$ implies $a_{\lambda} m=0$ for all $\lambda$, thus $a_{\lambda} \in \operatorname{Ann}_{A(g)}(m)$.

Let $\mathcal{L}_{g}$ be the collection of left ideals of $A(g)$ defined by:

$$
\mathcal{L}_{g}=\left\{\operatorname{Ann}_{A(g)}(m) \mid m \in M, M \in \mathcal{C}_{g}\right\} .
$$

For each $J \in \mathcal{L}_{g}$, we denote $J_{\lambda}=J \cap(A(g))_{\lambda}$. Then $J \supseteq \oplus_{\lambda} J_{\lambda}$. Note that $\oplus_{\lambda} J_{\lambda}$ is a graded left ideal of $U_{g}(V)$. (In general, $J$ is not graded.)

Lemma (1) For each $J \in \mathcal{L}_{g}, A(g)_{n}=J_{n}$ if $n \in \frac{1}{K} \mathbb{Z}$ is sufficiently small.

(2) Each $A(g)_{n} / J_{n}$ is finite dimensional.

(3) For $J_{1}, J_{2} \in \mathcal{L}_{g}$, there exists $J \in \mathcal{L}_{g}$ such that $J \subset J_{1} \cap J_{2}$.

(4) For any $a \in A(g)$ and $J \in \mathcal{L}_{g}$ there exists $I \in \mathcal{L}_{g}$ such that $I a \subset J$.

(5) $\cap_{J \in \mathcal{L}_{g}} J=\{0\}$.

(6) Every $J \in \mathcal{L}_{g}$ contains a graded left ideal $I \in \mathcal{L}_{g}$.

Proof: For convenience we write $J(m)=\operatorname{Ann}_{A(g)}(m)$ for $m \in M$ and $M \in \mathcal{C}_{g}$.

(1). We assume, at first, that $J=J(m)$ for a homogeneous element $m \in M_{\lambda}$ and $M \in \mathcal{C}_{g}$. Consider the $A(g)$-module homomorphism:

$$
\begin{array}{cl}
A(g) & \rightarrow A(g) m \\
a & \mapsto a m .
\end{array}
$$


Then $A(g) m$ is a $g$-twisted $V$-submodule of $M$ by Lemma 2.3. Since $(A(g) m)_{\lambda+n}=$ $A(g)_{n} m$ where $n \in \frac{1}{K} \mathbb{Z}$ and $(A(g) m)_{n+\lambda}=0$ for $n \in \frac{1}{K} \mathbb{Z}$ sufficiently small. Thus we must have $A(g)_{n} \subset J_{n}$ for $n \in \frac{1}{K} \mathbb{Z}$ and sufficiently small.

In general, let $m=\sum_{\mu} m_{\mu}$ with $m_{\mu} \in M_{\mu}$ with distinct $\mu$ 's in $\frac{1}{K} \mathbb{Z}$. Since $J=$ $\operatorname{Ann}_{A(g)}(m) \supseteq \cap_{\mu} J\left(m_{\mu}\right)$. Note that $J_{\lambda} \supseteq \cap_{\mu} J\left(m_{\mu}\right)_{\lambda}$ for any $\lambda$. Thus we have a surjective linear map $A(g)_{\lambda} / \cap_{\mu} J\left(m_{\mu}\right)_{\lambda} \rightarrow A(g)_{\lambda} / J_{\lambda}$ and $(A(g))_{\lambda} / \cap_{\mu} J\left(m_{\mu}\right)_{\lambda} \subseteq$ $\oplus_{\mu}(A(g))_{\lambda} / J\left(m_{\mu}\right)_{\lambda}$. Now the result follows from the proof for homogeneous $m$ above and the fact that there are only finitely many nonzero $m_{\mu}$ 's. The proof of $(2)$ is also included in this proof.

(3) Let $m_{i} \in M^{i}$ such that $J_{i}=\operatorname{Ann}_{A(g)}\left(m_{i}\right)$ for $i=1,2$. Consider the module $M=M^{1} \oplus M^{2}$ and $m=m_{1}+m_{2}$. Then $a \in \operatorname{Ann}_{A(g)}(m)$ if and only if $a \in J_{1} \cap J_{2}$. That is, $J_{1} \cap J_{2}=\operatorname{Ann}_{A(g)}(m) \in \mathcal{L}_{g}$.

(4) Let $J=\operatorname{Ann}_{A(g)}(m)$ with $m \in M$. For any $a \in A(g)$, set $I=\operatorname{Ann}_{A(g)}(a m) \in \mathcal{L}_{g}$. It is obvious that $I a \subset J$.

(5) If $a \in \cap_{J \in \mathcal{L}_{g}} J$, then $\rho_{M}(a)=0$ for all $M$ in $\mathcal{C}_{g}$. Therefore $a=0$ in $A(g)$ by the definition of the algebra $A(g)$ (see 2.4).

(6) Let $J=\operatorname{Ann}_{U_{g}(V)} m$ for some $m \in M$ for a $g$-twisted $V$-module $M$. Let $m=$ $\sum_{i=1}^{s} m_{i}$ with $m_{i} \in M_{\lambda_{i}}$ as homogeneous components. We have $J \supseteq \cap_{i=1}^{s} \operatorname{Ann}_{A(g)}\left(m_{i}\right)$. Here $\cap_{i=1}^{s} \operatorname{Ann}_{A(g)}\left(m_{i}\right)=\operatorname{Ann}_{A(g)}\left(\left(m_{1}, \ldots, m_{s}\right)\right)$, with $\left(m_{1}, \ldots, m_{s}\right) \in \bigoplus_{i=1}^{s} A(g) m_{i}$, is a graded left ideal and in $\mathcal{L}_{g}$.

Theorem The collection $\mathcal{L}_{g}$ forms a basis at 0 of a linear (Hausdorff) topology on $A(g)$ such that the topology defined earlier on $A(g)$ coincides with this topology. In particular, the graded left ideals in $\mathcal{L}_{g}$ also form a basis at 0 of this linear topology.

Proof: For each $M$ in $\mathcal{C}_{g}$, the pointwise convergence topology on $\operatorname{End}(M)$, which is a topological group which discrete topology on $M$, has a basis at 0 consists of sets

$$
W(m)=\{f \in \operatorname{End}(M) \mid f(m)=0\}
$$

for any $m \in M$ and the finite intersection of these sets. Since the topology on $A(g)$ defined earlier is the weakest topology on $A(g)$ such that $\rho_{M}: A(g) \rightarrow \operatorname{End}(M)$ is continues, then $\rho_{M}^{-1}(W(m))$ with $m \in M$ and $M$ running through $\mathcal{C}_{g}$ generates a basis of this topology at 0 on $A(g)$. However, by definition one sees that $\rho_{M}^{-1}(W(m))=$ $\operatorname{Ann}_{A(g)}(m)$. Therefore $\mathcal{L}_{g}$ is a basis of this topology.

It is a direct consequence of Lemma 2.6(5) that the topology is Hausdorff. By Lemma 2.6(6), the collection of graded left ideals in $\mathcal{L}_{g}$ also form a basis of this topology at 0 .

Corollary With the topology defined above the left and right multiplications by an element of $A(g)$ are continuous linear maps. 
Proof: Fix $a \in A(g)$. Clearly we have $a J \subset J$ for any $J \in \mathcal{L}_{g}$. This shows that the left multiplication by $a$ on $A(g)$ is continuous. By Lemma $2.6(4)$ there exists $I \in \mathcal{L}_{g}$ so that $I a \subset J$, i.e., the right multiplication by $a$ is continuous.

2.7. Extending the category $\mathcal{C}_{g}$ by the topology on $A(g)$. For each $A(g)$-module $M$, we define

$$
\mathcal{F}(M)=\left\{m \in M \mid J m=0 \text { for some } J \in \mathcal{L}_{g}\right\} .
$$

First of all, by Lemma 2.6(3), $\mathcal{F}(M)$ is vector subspace of $M$. Further by Lemma 2.6(4), we see that for any $m \in \mathcal{F}(M)$, then am $\in \mathcal{F}(M)$. Therefore $\mathcal{F}(M)$ is an $A(g)$ submodule of $M$. Following the definition, we can see $\mathcal{F}(\mathcal{F}(M))=\mathcal{F}(M)$. In particular by the definition of $\mathcal{L}_{g}$, we also have $\mathcal{F}(M)=M$ for all $M$ in $\mathcal{C}_{g}$.

Note that $A(g)$ is an associative algebra and is equipped with the topology defined above. We say an $A(g)$-module $M$ is continuous if the map $\pi: A(g) \times M \rightarrow M$ is continuous. All modules are equipped with discrete topology. However, an $A(g)$ module $M$ is continuous if and only if $\mathcal{F}(M)=M$. Indeed, all modules $M$ such that $\mathcal{F}(M)=M$ is continuous since for each $m \in M,(1+J) \times\{m\}$ is an open set in $A(g) \times M$ and $(1+J) m=m$. Here $J \in \mathcal{L}_{g}$ and $J m=0$. Conversely, if $M$ is continuous, $\pi^{-1}(0)$ is an open set in $A(g) \times M$. For any $m \in M$, since $(0, m) \in \pi^{-1}(0)$, there must be a $J \in \mathcal{L}_{g}$ such that $J \times\{m\} \subseteq \pi^{-1}(0)$. This shows that $J m=0$.

We now define $\overline{\mathcal{C}}_{g}$ to be the full subcategory of $A(g)$-modules $M$ such that $\mathcal{F}(M)=$ $M$. Therefore $\mathcal{C}_{g}$ is a full subcategory of $\overline{\mathcal{C}}_{g}$. We use $\mathcal{I}: \overline{\mathcal{C}}_{g} \rightarrow A(g)$-Mod to denote the natural embedding functor. It is straightforward from the definition to verify that $A(g)$ submodules and quotient of modules in $\overline{\mathcal{C}}_{g}$ are still in $\overline{\mathcal{C}}_{g}$. Thus for each $A(g)$-module $M, \mathcal{F}(M)$ is the unique maximal $A(g)$-submodule of $M$ contained in $\overline{\mathcal{C}}_{g}$. Furthermore, if $f: M \rightarrow N$ is a homomorphism of $A(g)$-modules for any two $A(g)$-modules $N$ and $M$, then $\mathcal{F}(M) \subseteq \mathcal{F}(N)$. Therefore we see that $\mathcal{F}: A(g)$-Mod $\rightarrow \overline{\mathcal{C}}_{g}$ is a functor.

Lemma (1) $(\mathcal{I}, \mathcal{F})$ is an adjoint pair of functors.

(2) $\overline{\mathcal{C}}_{g}$ is an Abelian category and closed under the direct limits of $A(g)$-modules.

Proof: (1) Let $M \in \overline{\mathcal{C}}_{g}$ and $N$ any $A(g)$-module. Any $f \in \operatorname{Hom}_{A(g)}(\mathcal{I} M, N)$ if and only if $f(M) \subseteq \mathcal{F}(N)$. The verification of functorial property is routine.

(2) All other conditions of an Abelian category are straightforward. We only verify that direct limits of $A(g)$-modules in $\overline{\mathcal{C}}_{g}$ are still in $\overline{\mathcal{C}}_{g}$. Let $\left(M_{\alpha}, f_{\alpha, \beta}\right)_{\alpha, \beta \in I}$ be a direct system. Let $M$ be the direct limit in $A(g)$-Mod with maps $f_{\alpha}: M_{\alpha} \rightarrow M$. Note that as $A(g)$-module, $M$ is generated by the submodules $f_{\alpha}\left(M_{\alpha}\right)$ for all $\alpha \in I$. Since $M_{\alpha}$ is in $\overline{\mathcal{C}}_{g}$, so is $f_{\alpha}\left(M_{\alpha}\right)$. Thus $f_{\alpha}\left(M_{\alpha}\right) \subseteq \mathcal{F}(M)$ for all $\alpha \in I$. But $\mathcal{F}(M)$ is a submodule of $M$. Hence $\mathcal{F}(M)=M$ and $M$ is in $\overline{\mathcal{C}}_{g}$. 
Theorem Let $M$ be any $A(g)$-module and $m \in \mathcal{F}(M)$. Then $A(g) m$ is in $\mathcal{C}_{g}$. In particular, every module in $\overline{\mathcal{C}}_{g}$ is a union of submodules in $\mathcal{C}_{g}$ and direct sum of $L(0)$ eigenspaces.

Proof: Since $m \in \mathcal{F}(M)$ there exists $J \in \mathcal{L}_{g}$ such that $J m=0$. Let $W \in \mathcal{C}_{g}$ and $w \in W$ with $J=\operatorname{Ann}_{A(g)}(w)$. Clearly, $A(g) w$ is a $g$-twisted $V$-submodule of $W$. Note that the map from $A(g) w$ to $A(g) m$ by sending aw to am is well defined and an $A(g)$ module homomorphism. Thus $A(g) m$ is a $g$-twisted $V$-module, that is, $A(g) m \in \mathcal{C}_{g}$. The other assertions in the theorem are clear now.

Corollary (1) A module $M$ in $\overline{\mathcal{C}}_{g}$ is in $\mathcal{C}_{g}$ if and only if for each $\lambda \in \mathbb{C}, M_{\lambda}$ is finite dimensional and $M_{\lambda-n}=0$ for $n \in \frac{1}{K} \mathbb{Z}$ with $n>>0$.

(2) $\operatorname{Ext}_{\mathcal{C}_{g}}^{1}(M, N)=\operatorname{Ext}_{\overline{\mathcal{C}}_{g}}^{1}(M, N)$ for all $M, N$ in $\mathcal{C}_{g}$.

(3) Every simple module in $\overline{\mathcal{C}}_{g}$ is in $\mathcal{C}_{g}$.

Proof: (1) Let $M$ be any module in $\overline{\mathcal{C}_{g}}$. We can define

$$
Y_{M}(v, z)=\sum_{n \in \frac{1}{K} \mathbb{Z}} v_{n} z^{-n-1}
$$

in $\operatorname{End}(M)\{z\}$ for each $v \in V$. Here $v_{n}$ is the image of $v \otimes t^{n}$ in $\operatorname{End}(M)$. Note that all conditions for a $g$-twisted $V$-module except Eq. (1) and Eq. (5) are satisfied. Now we show that for so defined $Y_{M}(v, z)$, the Jacobi identity is also satisfied. We only need to verify Eq. (10). For each $u \in V^{r}$ and $v \in V$, we denote by

$$
\begin{gathered}
f(X)=\sum_{m \geq 0}(-1)^{m}\left(\begin{array}{c}
p \\
m
\end{array}\right)\left(u_{p+s-m} v_{t+m}-(-1)^{p} v_{p+t-m} u_{s+m}\right) X^{m} \\
g(X)=\sum_{m \geq 0}\left(\begin{array}{c}
s \\
m
\end{array}\right)\left(u_{p+m} v\right)_{s+t-m} X^{m}
\end{gathered}
$$

to be two elements of $C$. We will use $f(X)$ and $g(X)$ to denote their images in $A(g)$ as well. Since, for any $g$-twisted $V$-module $W$, we have $f(X) w=g(X) w$ for all $w \in W$ by the Jacobi identity (10), we have $f(X)=g(X)$ in $A(g)$. So we have $f(X) m=g(X) m$ for all $m \in M$ since $M$ is an $A(g)$-module. This only shows that $f(X)=g(X)$ as elements of $A(g)$, but there no reason to believe that the actions of $f(X)$ on $M$ is the same as the "limit" as required by the Jacobi identity. Thus we have to show that for any $m \in M$,

$$
f(X) m=\sum_{i=0}^{n}(-1)^{i}\left(\begin{array}{c}
p \\
i
\end{array}\right)\left(u_{p+s-i} v_{t+i}-(-1)^{p} v_{p+t-i} u_{s+i}\right) m
$$


and

$$
g(X) m=\sum_{i=0}^{n}\left(\begin{array}{l}
s \\
i
\end{array}\right)\left(u_{p+i} v\right)_{s+t-i} m
$$

for sufficiently large $n$. Set

$$
f_{n}(X)=\sum_{i=0}^{n}(-1)^{i}\left(\begin{array}{l}
p \\
i
\end{array}\right)\left(u_{p+s-i} v_{t+i}-(-1)^{p} v_{p+t-i} u_{s+i}\right) X^{i}
$$

and

$$
g_{n}(X)=\sum_{i=0}^{n}\left(\begin{array}{l}
s \\
i
\end{array}\right)\left(u_{p+i} v\right)_{s+t-i} X^{i} .
$$

Here we have to use the fact that all modules in $\overline{\mathcal{C}}_{g}$ are continuous. Since $M$ is in $\overline{\mathcal{C}}_{g}$, there exists $J \in \mathcal{L}_{g}$ such that $J m=0$. Then $J=\operatorname{Ann}_{A(g)}(w)$ for some $W$ in $\mathcal{C}_{g}$ and $w \in W$. Since $W$ is $g$-twisted, there exists $n_{0}$ such that $f(X) w=f_{n}(X) w$ for all $n \geq n_{0}$, i.e., $f(X)-f_{n}(X) \in J$. Thus we have $f(X) w=f_{n}(X) w$. Equality $g(X) m=g_{n}(X) m$ for sufficiently large $n$ is proved in the same way.

Finally, in order for $M$ in $\overline{\mathcal{C}}_{g}$ to be in $\mathcal{C}_{g}$, one only needs the condition Eq. (1). But this is assumed in the corollary.

(2) Let $0 \rightarrow N \rightarrow E \rightarrow M \rightarrow 0$ be an extension in $\overline{\mathcal{C}}_{g}$. Since $E$ is in $\overline{\mathcal{C}}_{g}$, by (1), we have $E \in \mathcal{C}_{g}$. This shows that every extension in $\overline{\mathcal{C}}_{g}$ is actually an extension in $\mathcal{C}_{g}$. Since $\mathcal{C}_{g}$ is a full subcategory of $\overline{\mathcal{C}}_{g}$, two extensions in $\mathcal{C}_{g}$ are equivalent $\mathcal{C}_{g}$ if and only if they are equivalent in $\overline{\mathcal{C}}_{g}$. (3) follows from the Theorem since $A(g) m$ is a submodule of $M$ and in $\mathcal{C}_{g}$,

2.8. Construction of modules in $\overline{\mathcal{C}}_{g}$. Let $M$ be a $\mathbb{C}$-vector space. Then space $\operatorname{Hom}_{\mathbb{C}}(A(g), M)$ has an $A(g)$-module structure defined in the following way:

$$
(a f)(x)=f(x a)
$$

for $f \in \operatorname{Hom}_{\mathbb{C}}(A(g), M)$ and $a \in A(g)$. One can easily see that

$$
(a b f)(x)=f(x a b)=(b f)(x a)=(a(b f))(x) .
$$

We define $\mathcal{H}(M)=\mathcal{F}\left(\operatorname{Hom}_{\mathbb{C}}(A(g), M)\right)$ then $\mathcal{H}(M) \in \overline{\mathcal{C}}_{g}$.

There is a natural $\mathbb{C}$-linear map ev: $\mathcal{H}(M) \rightarrow M$ defined by $\operatorname{ev}(f)=f(1)$ for all $f \in \mathcal{H}_{\tilde{\phi}}(M)$. If $N$ is another vector space $\phi: N \rightarrow M$ is a linear map, the natural induced map $\tilde{\phi}: \operatorname{Hom}_{\mathbb{C}}(A(g), N) \rightarrow \operatorname{Hom}_{\mathbb{C}}(A(g), M)$ is an $A(g)$-module homomorphism, which further induces an $A(g)$-module homomorphism $\mathcal{H}(\phi): \mathcal{H}(N) \rightarrow \mathcal{H}(M)$. Thus one can verify that $\mathcal{H}$ defines a functor $\mathbb{C}$-Vect $\rightarrow \overline{\mathcal{C}}_{g}$, where $\mathbb{C}$-Vect is the category of complex vector spaces. If $\mathcal{G}: \overline{\mathcal{C}}_{g} \rightarrow \mathbb{C}$-Vect is the forgetful functor, then

Lemma $\mathcal{H}$ is a left exact functor from the category of vector spaces to $\overline{\mathcal{C}}_{g}$. Furthermore, $(\mathcal{G}, \mathcal{H})$ is an adjoint pair. 
Proof: As the right adjoint to the exact functor $\mathcal{I}, \mathcal{F}$ is obvious left exact. It is well known that the functor $\operatorname{Hom}_{\mathbb{C}}(A(g), \cdot)$ is left exact. So $\mathcal{H}$ is a composition of two left exact functors and is left exact.

The adjoint pair follows from the following isomorphism of vector spaces for any $N$ in $\overline{\mathcal{C}}_{g}$ and vector space $M$ :

$$
\begin{aligned}
\operatorname{Hom}_{\mathbb{C}}(N, M) & =\operatorname{Hom}_{\mathbb{C}}\left(A(g) \otimes_{A(g)} N, M\right) \\
& \cong \operatorname{Hom}_{A(g)}\left(N, \operatorname{Hom}_{\mathbb{C}}(A(g), M)\right)=\operatorname{Hom}_{A(g)}(N, \mathcal{H}(M)) .
\end{aligned}
$$

Here the second isomorphism comes from the adjointness of the "tensor functor" and "Hom functor" while the last equality follows from the fact that $N$ is in $\overline{\mathcal{C}}_{g}$. Naturality of the isomorphism is also routine to check.

Remark. Since both $\mathcal{F}$ and $\mathcal{H}$ have exact left adjoint functors, they send injective modules to injective modules. In particular, $\mathcal{H}(M)$ is an injective module in $\overline{\mathcal{C}}_{g}$ for $M \in \mathbb{C}$-Vect.

Let $M \in \overline{\mathcal{C}}_{g}$. Define a linear map $\phi: M \rightarrow \operatorname{Hom}_{\mathbb{C}}(A(g), M)$ by $\phi(m)=f_{m}: a \mapsto a m$ for $m \in M$ and $a \in A(g)$. Then for $a, b \in A(g)$ we have

$$
\phi(a m)(b)=f_{a m}(b)=b(a m)=f_{m}(b a)=\left(a f_{m}\right)(b)=(a \phi(m))(b) .
$$

This shows that $\phi$ is an $A(g)$-module homomorphism. Thus $\phi(M) \subset \mathcal{F}\left(\operatorname{Hom}_{\mathbb{C}}(A(g), M)\right)$ $=\mathcal{H}(M)$ since $\phi(M)$ is in $\overline{\mathcal{C}}_{g}$. Next we show that $\phi$ is injective. If $\phi(m)=0$ then $\phi(m)(a)=a m=0$ for all $a \in A(g)$. In particular, $\phi(m)(1)=m=0$. Note that the functor $\mathcal{H}$ preserves the arbitrary direct sums. Then $\mathcal{H}(M)=\oplus_{b \in B} \mathcal{H}(\mathbb{C} b)$ with $B \subseteq M$ being a $\mathbb{C}$-basis. Thus we have proved

Theorem $\mathcal{C}_{g}$ has enough injective objects and every injective module is a direct summand of the direct sum of copies of $\mathcal{H}(\mathbb{C})$.

2.9. Proposition If $\mathcal{C}_{g}$ is semisimple, then all modules in $\overline{\mathcal{C}}_{g}$ are also semisimple. Proof: Let $M$ be a module in $\overline{\mathcal{C}}_{g}$. For any $m \in M$, there exists a submodule $N \subseteq M$ such that $N$ is in $\mathcal{C}_{g}$ and $m \in N$. Since $N$ is a direct sum of simple modules in $\mathcal{C}_{g}$, we have shown that $M$ is the sum of all simple submodules in $\mathcal{C}_{g}$. Thus $M$ is a direct sum of simple modules in $\mathcal{C}_{g}$.

\section{Induction for a subalgebra}

3.1. Let $V^{\prime}$ be a vertex operator subalgebra of $V$ such that $g\left(V^{\prime}\right)=V^{\prime}$. Let $g^{\prime}=\left.g\right|_{V^{\prime}}$ and $o\left(g^{\prime}\right)=K^{\prime}$. Then $K^{\prime} \mid K$ and each $g$-twisted $V$-module $M$ when restricted to $V^{\prime}$ is a 
$g^{\prime}$-twisted $V^{\prime}$-module. Let $\mathcal{C}_{g^{\prime}}^{\prime}$ be the category all $g^{\prime}$-twisted $V^{\prime}$-module. Then we have a restriction functor

$$
\operatorname{Res}_{\overline{\mathcal{C}}_{g^{\prime}}^{\prime}}^{\overline{\overline{\mathcal{O}}_{g}}}: \mathcal{C}_{g} \rightarrow \mathcal{C}_{g^{\prime}}^{\prime}
$$

Consider the enveloping algebras $A(g)$ and $A\left(g^{\prime}\right)$. The natural embedding $\bar{V}^{\prime}=$ $\oplus_{r=0}^{K^{\prime}-1}\left(V^{\prime}\right)^{r} \otimes t^{r / K^{\prime}} \mathbb{C}\left[t, t^{-1}\right] \rightarrow \bar{V}$ induces an algebra homomorphism $\psi: T\left(\bar{V}^{\prime}\right) \rightarrow T(\bar{V})$ where $\left(V^{\prime}\right)^{r}=\left\{v \in V^{\prime} \mid g^{\prime} v=e^{r 2 \pi i / K^{\prime}} v\right\}$. For each $M \in \mathcal{C}_{g}$ the composition of the algebra homomorphism $\rho_{M}: T(\bar{V}) \rightarrow \operatorname{End}_{\mathbb{C}}(M)$ with $\psi$ produces an algebra homomorphism $\rho_{M}^{\prime}: T\left(\bar{V}^{\prime}\right) \rightarrow \operatorname{End}_{\mathbb{C}}(M)$. Since $\left.M\right|_{V^{\prime}}$ is a module for $U_{g^{\prime}}\left(V^{\prime}\right)$ the kernel $I^{\prime}$ of $T\left(\bar{V}^{\prime}\right) \rightarrow U_{g^{\prime}}\left(V^{\prime}\right) \rightarrow 0$ is contained in $\operatorname{Ker} \rho_{M}^{\prime}$ and $\psi\left(\operatorname{Ker} \rho_{M}^{\prime}\right) \subset \operatorname{Ker} \rho_{M}$. Thus $\psi\left(I^{\prime}\right) \subset I=\cap_{M \in \mathcal{C}_{g}} \operatorname{Ker} \rho_{M}$ and $\psi$ induces an algebra homomorphism $U_{g^{\prime}}\left(V^{\prime}\right) \rightarrow U_{g}(V)$, which is still denoted by $\psi$.

Theorem With the above assumption, the algebra homomorphism $\psi: U_{g^{\prime}}\left(V^{\prime}\right) \rightarrow$ $U_{g}(V)$ induces a homomorphism $\bar{\psi}: A\left(g^{\prime}\right) \rightarrow A(g)$ of associative algebras such that for each $M \in \mathcal{C}_{g}$, $\operatorname{Res}_{\overline{\mathcal{C}}_{g^{\prime}}^{\prime}}^{\overline{\overline{\mathcal{C}}_{g}}}(M)$ as $A\left(g^{\prime}\right)$-module factors through $A(g)$, that is, $A\left(g^{\prime}\right) \rightarrow$ $A(g) \rightarrow \operatorname{End}_{\mathbb{C}}(M)$.

Proof: The homomorphism $\psi: U_{g^{\prime}}\left(V^{\prime}\right) \rightarrow U_{g}(V)$ induces an algebra homomorphism $\psi[X]: U_{g^{\prime}}\left(V^{\prime}\right)[[X]] \rightarrow U_{g}(V)[[X]]$. It follows from the definition of $C_{M}(g)$ in 2.4, that $\psi[X]\left(C_{\left.M\right|_{V^{\prime}}}\right) \subseteq C_{M}$. Since $\left.M\right|_{V^{\prime}}$ is in $\mathcal{C}_{g^{\prime}}^{\prime}\left(V^{\prime}\right)$ for each $M$ in $\mathcal{C}_{g}(V), \psi[X]$ induces an algebra homomorphism $\bar{\psi}: A\left(g^{\prime}\right) \rightarrow A(g)$. The conditions on the module structures in the theorem are clear from the construction of the algebra homomorphism $\bar{\psi}$.

3.2. Let $\mathcal{L}_{g^{\prime}}^{\prime}$ be the corresponding collection of left ideals defining the topology on $A\left(g^{\prime}\right)$ as in 2.6 .

Lemma For any $J \in \mathcal{L}_{g}$ there exists $J^{\prime} \in \mathcal{L}_{J^{\prime}}^{\prime}$ such that $\bar{\psi}\left(J^{\prime}\right) \subset J$.

Proof: Let $m \in M$ with $M \in \mathcal{C}_{g}$ and $J=\operatorname{Ann}_{A(g)}(m)$. Set $J^{\prime}=\operatorname{Ann}_{A\left(g^{\prime}\right)}(m)$. Then $J^{\prime} \in \mathcal{L}_{g^{\prime}}^{\prime}$. Now the result follows.

Let $\mathcal{C}_{g^{\prime}}^{\prime}$ be the corresponding category for $V^{\prime}$ and $g^{\prime}$. For any $A\left(g^{\prime}\right)$-module $M^{\prime}$, let $\mathcal{F}^{\prime}\left(M^{\prime}\right)$ be the submodule of $M^{\prime}$ for $A\left(g^{\prime}\right)$. We will use $\overline{\mathcal{C}}_{g^{\prime}}^{\prime}$ to denote the category of $A\left(g^{\prime}\right)$-modules $M^{\prime}$ such that $\mathcal{F}^{\prime}\left(M^{\prime}\right)=M^{\prime}$. For each $A(g)$-module $M,\left.M\right|_{A\left(g^{\prime}\right)}$ is the $A(g)$-module via $\bar{\phi}$. The lemma implies that $\mathcal{F}(M) \subseteq \mathcal{F}^{\prime}\left(\left.M\right|_{A\left(g^{\prime}\right)}\right)$. In particular, $\left.M\right|_{A(g)}$ is in $\overline{\mathcal{C}}_{g^{\prime}}^{\prime}$ if $M$ is in $\overline{\mathcal{C}}_{g}$. We thus defines a functor $\overline{\mathcal{C}}_{g} \rightarrow \overline{\mathcal{C}}_{g^{\prime}}^{\prime}$. We will still use $\operatorname{Res}_{\overline{\mathcal{C}}_{g^{\prime}}^{\prime}}^{\overline{\mathcal{C}}_{g}}$ to denote this functor if $V$ and $V^{\prime}$ are understood from the context.

Proposition The restriction functor $\operatorname{Res}_{\overline{\mathcal{C}}_{g^{\prime}}^{\prime}}^{\overline{\mathcal{C}}_{g}}: \overline{\mathcal{C}}_{g} \rightarrow \overline{\mathcal{C}}_{g^{\prime}}^{\prime}$ is exact. 
3.3. We consider $A(g)$ as a left $A\left(g^{\prime}\right)$-module and a right $A(g)$-module, i.e., $A(g)$ is a $A\left(g^{\prime}\right)$ - $A(g)$-bimodule. For each $M \in \overline{\mathcal{C}}_{g^{\prime}}^{\prime}, \operatorname{Hom}_{A\left(g^{\prime}\right)}(A(g), M)$ is a left $A(g)$-module with the action $(a f)(x)=f(x a)$ for $a, x \in A(g)$ and $f \in \operatorname{Hom}_{A\left(g^{\prime}\right)}(A(g), M)$. We define

$$
\operatorname{Ind}_{\overline{\mathcal{C}}_{g^{\prime}}^{\prime}}^{\overline{\mathcal{C}}_{g}}(M)=\mathcal{F}\left(\operatorname{Hom}_{A\left(g^{\prime}\right)}(A(g), M)\right)
$$

Then $\operatorname{Ind}_{\overline{\mathcal{C}}_{g^{\prime}}^{\prime}}^{\overline{\mathcal{C}}_{g}}(M)$ is a module in $\overline{\mathcal{C}}_{g}$.

If $M$ and $N$ are in $\overline{\mathcal{C}}_{g^{\prime}}^{\prime}$ and $\phi: M \rightarrow N$ is a homomorphism of $g^{\prime}$-twisted $V^{\prime}$-modules, then

$$
\begin{array}{ccc}
\tilde{\phi}: \operatorname{Hom}_{A\left(g^{\prime}\right)}(A(g), M) & \rightarrow & \operatorname{Hom}_{A\left(g^{\prime}\right)}(A(g), N) \\
f & \mapsto & \phi \circ f
\end{array}
$$

is a homomorphism of $A(g)$-modules. Indeed, for $f \in \operatorname{Hom}_{A\left(g^{\prime}\right)}(A(g), M)$, and $a, x \in$ $A(g)$, we have

$$
(\tilde{\phi}(a f))(x)=(\phi \circ(a f))(x)=\phi(f(x a))=(\phi \circ f)(x a)=(a \tilde{\phi}(f))(x),
$$

that is, $\tilde{\phi}(a f)=a \tilde{\phi}(f)$. Also, for $a^{\prime} \in A\left(g^{\prime}\right)$

$$
(\tilde{\phi}(f))\left(\bar{\psi}\left(a^{\prime}\right) x\right)=\phi\left(f\left(\bar{\psi}\left(a^{\prime}\right) x\right)\right)=\phi\left(a^{\prime} f(x)\right)=a^{\prime} \phi(f(x))=a^{\prime}(\tilde{\phi}(f)(x)),
$$

i.e., $\tilde{\phi}(f) \in \operatorname{Hom}_{A\left(g^{\prime}\right)}(A(g), N)$. Then the map $f \rightarrow \phi \circ f$ induces a homomorphism

$$
\operatorname{Ind}_{\overline{\mathcal{C}}_{g^{\prime}}}^{\overline{\mathcal{C}}_{g}}(\phi): \operatorname{Ind}_{\overline{\mathcal{C}}_{g^{\prime}}^{\prime}}^{\overline{\mathcal{C}}_{g}}(M) \rightarrow \operatorname{Ind}_{\overline{\mathcal{C}}_{g^{\prime}}^{\prime}}^{\overline{\mathcal{C}}_{g}}(N)
$$

in $\overline{\mathcal{C}_{g}}$. We thus define a functor $\operatorname{Ind}_{\overline{\mathcal{C}}_{g^{\prime}}^{\prime}}^{\overline{\mathcal{C}}_{g}}: \overline{\mathcal{C}}_{g^{\prime}}^{\prime} \rightarrow \overline{\mathcal{C}}_{g}$, which we call the induction functor.

3.4. For each $M \in \overline{\mathcal{C}}_{g^{\prime}}^{\prime}$, there is an evaluation map

$$
e v: \operatorname{Ind}_{\overline{\mathcal{C}}_{g^{\prime}}^{\prime}}^{\overline{\mathcal{C}}_{g}}(M) \rightarrow M
$$

defined by $e v(f)=f(1)$.

Lemma $e v$ is a homomorphism of $A\left(g^{\prime}\right)$-modules when $\operatorname{Ind}_{{\overline{\mathcal{C}^{\prime}}}_{g^{\prime}}}^{\overline{\mathcal{C}}_{g}}(M)$ is restricted to $A\left(g^{\prime}\right)$ via $\bar{\psi}$.

Proof: For $a^{\prime} \in A\left(g^{\prime}\right)$ and $f \in \operatorname{Ind}_{\overline{\mathcal{C}}_{g^{\prime}}}^{\overline{\mathcal{C}}_{g}}(M)$, we have

$$
e v\left(a^{\prime} f\right)=\left(a^{\prime} f\right)(1)=f\left(1 \bar{\psi}\left(a^{\prime}\right)\right)=f\left(\bar{\psi}\left(a^{\prime}\right) 1\right)=a^{\prime} f(1)=a^{\prime} e v(f),
$$

as desired. 
Lemma For any $M \in \overline{\mathcal{C}}_{g}$ we have ev : $\operatorname{Hom}_{U_{g}(V)}\left(U_{g}(V), M\right) \simeq M$. Moreover, if $M \in \mathcal{C}_{g}$ then $\operatorname{Ind}_{\overline{\mathcal{C}}_{g}}^{\overline{\mathcal{C}}_{g}}(M) \simeq M$.

Proof: We need to prove that $e v$ is one to one and onto in this case. If $f(1)=0$ then $f(a)=a f(1)=0$ for any $a \in U_{g}(V)$ thus $f=0$ and $e v$ is one to one. Let $m \in M$ and define $f_{m} \in \operatorname{Hom}_{U_{g}(V)}\left(U_{g}(V), M\right)$ by $f_{m}(a)=a m$. Then $f(1)=m$ and $e v$ is onto.

Theorem (Frobenius Reciprocity) For any $E \in \overline{\mathcal{C}}_{g}$ and $M \in \overline{\mathcal{C}}_{g^{\prime}}^{\prime}$ the natural map

$$
\Phi: \operatorname{Hom}_{A(g)}\left(E, \operatorname{Ind}_{\overline{\mathcal{C}}_{g^{\prime}}^{\prime}}^{\overline{\mathcal{C}}_{g}}(M)\right) \rightarrow \operatorname{Hom}_{A\left(g^{\prime}\right)}\left(\operatorname{Res}_{V^{\prime}}^{V}(E), M\right)
$$

defined by $\phi \mapsto e v \circ \phi$ is an isomorphism of vector spaces.

Proof: First $e v \circ \phi=\Phi(\phi)$ is a composition of $A\left(g^{\prime}\right)$-homomorphisms and thus is a homomorphism in $\overline{\mathcal{C}}_{g^{\prime}}^{\prime}$. We only need to construct the inverse $\Phi^{-1}$. For each $\phi^{\prime} \in$ $\operatorname{Hom}_{A\left(g^{\prime}\right)}\left(\operatorname{Res}_{\overline{\mathcal{C}}_{g^{\prime}}^{\prime}}^{\overline{\overline{\mathcal{A}}_{g}}}(E), M\right)$ define

$$
\phi: E \rightarrow \operatorname{Hom}_{\mathbb{C}}(A(g), M)
$$

such that

$$
(\phi(e))(x)=\phi^{\prime}(x e)
$$

for $e \in E$ and $x \in A(g)$. In fact $\phi$ is the composition:

$$
\begin{array}{cccc}
A(g) & \rightarrow E & \rightarrow & M \\
x & \mapsto x e & \mapsto \phi^{\prime}(x e) .
\end{array}
$$

Then $\phi(e)$ is a homomorphism of $A\left(g^{\prime}\right)$-modules. Since for $x^{\prime} \in A\left(g^{\prime}\right)$

$$
\phi(e)\left(\bar{\psi}\left(a^{\prime}\right) x\right)=\phi^{\prime}\left(\bar{\psi}\left(a^{\prime}\right) x e\right)=a^{\prime} \phi^{\prime}(x e)=a^{\prime} \phi(e)(x) .
$$

Thus we have a map $\phi: E \rightarrow \operatorname{Hom}_{A\left(g^{\prime}\right)}(A(g), M)$. Moreover $\phi$ is an $A(g)$-homomorphism:

$$
\phi(a e)(x)=\phi^{\prime}(x a e)=\phi^{\prime}(e)(x a)=(a \phi(e))(x) .
$$

Since $E \in \overline{\mathcal{C}}_{g}$ so is its image $\phi(E)$ in $\operatorname{Hom}_{A\left(g^{\prime}\right)}(A(g), M)$. Now by the definition of the functor $\mathcal{F}$ we have $\phi(E) \subset \operatorname{Ind}_{\overline{\mathcal{C}}_{g^{\prime}}^{\prime}}^{\overline{\mathcal{C}}_{g}}(M)$. We define $\Phi^{-1}\left(\phi^{\prime}\right)=\phi$. Clearly $\Phi^{-1}$ is a linear map.

To finish the proof, we need to show that $\Phi^{-1}$ is an inverse of $\Phi$. Let $f \in \operatorname{Hom}_{A(g)}\left(E, \operatorname{Ind}_{\overline{\mathcal{C}}_{g^{\prime}}^{\prime}}^{\overline{\mathcal{C}}_{g}}(M)\right)$ and $\phi^{\prime}=e v \circ f$. Then

$$
\phi(e)(x)=\phi^{\prime}(x e)=(e v \circ f)(x e)=f(x e)(1)=(x f(e))(1)=f(e)(x)
$$


for $e \in E$ and $x \in A(g)$. Thus $\phi(e)=f(e), \phi=f$ and $\Phi^{-1} \circ \Phi=i d$.

Conversely, if $\phi^{\prime} \in \operatorname{Hom}_{A\left(g^{\prime}\right)}\left(\operatorname{Res}_{\overline{\mathcal{C}}_{g^{\prime}}^{\prime}}^{\overline{\mathcal{C}}_{g}}(E), M\right)$ and $\phi=\Phi^{-1}\left(\phi^{\prime}\right)$, then

$$
\Phi(\phi)(e)=(e v \circ \phi)(e)=e v(\phi(e))=\phi(e)(1)=\phi(e)(1)=\phi^{\prime}(1 e)=\phi^{\prime}(e),
$$

i.e., $\Phi \circ \Phi^{-1}=i d$. This completes the proof.

Corollary (1) $\left(\operatorname{Res}_{\overline{\mathcal{C}}_{g^{\prime}}^{\prime}}^{\overline{\overline{\mathcal{C}}_{g}}}, \operatorname{Ind}_{\overline{\mathcal{C}}_{g^{\prime}}}^{\overline{\mathcal{C}_{g}}}\right)$ is an adjoint pair of functors.

(2) $\operatorname{Ind}_{\overline{\mathcal{C}}_{g^{\prime}}^{\prime}}^{\overline{\mathcal{C}}_{g}}$ is left exact and sends injective modules to injective modules.

Remark. For a module $M$ in $\overline{\mathcal{C}}_{g}$, define $\operatorname{Soc}_{\overline{\mathcal{C}}_{g}}(M)$, called the socle of $M$, to be the largest semisimple submodule of $M$ and $\operatorname{Hd}_{\mathcal{C}_{g}}(M)$, called the head of $M$, to be the largest semisimple quotient of $M$. If $M$ a semisimple module (need not be finite length) and $S$ is a simple module, we denote by $[M, S]$ the cardinality of the copies of $S$ appearing in the direct sum decomposition into simple modules. If $M$ is a simple module in $\overline{\mathcal{C}}_{g^{\prime}}$ and $N$ simple module in $\overline{\mathcal{C}}_{g}$, then the Frobenius reciprocity implies that

$$
\left[\operatorname{Hd}_{\mathcal{C}_{g^{\prime}}^{\prime}}\left(\operatorname{Res}_{\overline{\mathcal{C}}_{g^{\prime}}^{\prime}}^{\overline{\mathcal{C}_{g}}}(N)\right): M\right]=\left[\operatorname{Soc}_{\overline{\mathcal{C}}_{g}}\left(\operatorname{Ind}_{\overline{\mathcal{C}}_{g^{\prime}}^{\prime}}^{\overline{\mathcal{C}_{g}}}(M)\right): N\right]
$$

However, it is not clear that in the category $\mathcal{C}_{g}$, every nonzero module has a nonzero socle or quotient.

3.5. Transitivity. Let $V^{\prime \prime} \subset V^{\prime} \subset V$ be subalgebras of $V$ which are $g$-stable. Set $g^{\prime \prime}=\left.g^{\prime}\right|_{V^{\prime \prime}}$. We shall use the obvious notation $\overline{\mathcal{C}}_{g^{\prime \prime}}^{\prime \prime}$ for the corresponding category of $g^{\prime \prime}$-twisted $V^{\prime \prime}$-modules. Let $M \in \overline{\mathcal{C}}_{g^{\prime \prime}}^{\prime \prime}$ and define

$$
\xi_{M}: \operatorname{Ind}_{\overline{\mathcal{C}}_{g^{\prime \prime}}^{\prime \prime}}^{\overline{\mathcal{C}}_{g}}(M) \rightarrow \operatorname{Ind}_{{\overline{\mathcal{C}^{\prime}}}_{g^{\prime}}}^{\overline{\mathcal{C}}_{g}} \operatorname{Ind}_{\overline{\mathcal{C}}_{g^{\prime \prime}}^{\prime \prime \prime}}^{\overline{\mathcal{C}}_{g^{\prime}}^{\prime}}(M)
$$

by $\xi_{M}(f)=\bar{f}: A(g) \rightarrow \operatorname{Ind}_{\overline{\mathcal{C}}_{g^{\prime \prime}}^{\prime \prime}} \frac{\overline{\mathcal{C}}^{\prime}}{g^{\prime}} M$. The definition of $\bar{f}$ is as follows: for $x \in A(g), \bar{f}(x)$ is a map:

$$
\begin{aligned}
\bar{f}(x): \quad A\left(g^{\prime}\right) & \rightarrow \\
y & \mapsto f(\bar{\psi}(y) x) .
\end{aligned}
$$

Recall that $\bar{\psi}$ is the algebra homomorphism from $A\left(g^{\prime}\right)$ to $A(g)$.

Theorem $\xi_{M}$ is an isomorphism of $A(g)$-modules, and further induces an isomorphism of functors $\xi: \operatorname{Ind}_{\overline{\mathcal{C}}_{g^{\prime \prime}}}^{\overline{\mathcal{C}_{g}}} \rightarrow \operatorname{Ind}_{\overline{\mathcal{C}}_{g^{\prime}}}^{\overline{\mathcal{C}}_{g}} \operatorname{Ind}_{\overline{\mathcal{C}}_{g^{\prime \prime}}}^{\overline{\mathcal{C}}_{g^{\prime}}^{\prime \prime}}$. 
Proof: First we show that $\xi_{M}(f) \in \operatorname{Ind}_{\overline{\mathcal{C}}_{g^{\prime}}^{\prime}}^{\overline{\mathcal{C}}_{g}} \operatorname{Ind}_{\overline{\mathcal{C}}_{g^{\prime \prime}}^{\prime \prime}} \overline{\mathcal{C}}^{\prime}(M)$. Let $\psi^{\prime}$ and $\psi^{\prime \prime}$ be the induced algebra homomorphisms $\psi^{\prime}: A\left(g^{\prime \prime}\right) \rightarrow A\left(g^{\prime}\right)$ and $\psi^{\prime \prime}: A\left(g^{\prime \prime}\right) \rightarrow A(g)$, respectively. Then $\psi^{\prime \prime}=\bar{\psi} \circ \psi^{\prime}$. For $z \in A\left(g^{\prime \prime}\right)$ and $f \in \operatorname{Ind}_{\overline{\mathcal{C}}_{g^{\prime \prime}} g^{\prime \prime}}^{\overline{\mathcal{C}}^{\prime \prime}}(M)$, we have

$$
\bar{f}(x)\left(\psi^{\prime}(z) y\right)=f\left(\bar{\psi}\left(\psi^{\prime}(z) y\right) x\right)=f\left(\psi^{\prime \prime}(z) \bar{\psi}(y) x\right)=z f(\bar{\psi}(y) x)=z \bar{f}(x)(y),
$$

i.e., $\bar{f}(x)$ is an $A\left(g^{\prime \prime}\right)$-module homomorphism.

To see $\bar{f}(x) \in \operatorname{Ind}_{\overline{\mathcal{C}_{g^{\prime \prime}}}}^{{\overline{g^{\prime}}}^{\prime \prime}}(M)$, we need to show that there exists $J^{\prime} \in \mathcal{L}_{g^{\prime}}^{\prime}$ such that $J^{\prime} \bar{f}(x)=0$. Let $J \in \mathcal{L}_{g}$ such that $J f=0$ or equivalently $f(J)=0$. From Lemma 2.6 (4) there exists $I \in \mathcal{L}_{g}$ so that $I x \subset J$. By Lemma 3.2 there exists $J^{\prime} \in \mathcal{L}_{g^{\prime}}^{\prime}$ such that $\bar{\psi}\left(J^{\prime}\right) \subset I$. Thus

$$
\left(J^{\prime} \bar{f}(x)\right)(y)=\bar{f}(x)\left(y J^{\prime}\right)=f\left(\bar{\psi}\left(y J^{\prime}\right) x\right) \subset f(I x) \subset f(J)=\{0\},
$$

that is, $\bar{f}(x) \in \operatorname{Ind}_{\overline{\mathcal{C}}_{g^{\prime \prime}}^{\prime \prime \prime}}^{\overline{g^{\prime}}}(M)$.

For $a^{\prime} \in A\left(g^{\prime}\right)$, we have

$$
\bar{f}\left(\bar{\psi}\left(a^{\prime}\right) x\right)(y)=f\left(\bar{\psi}(y) \bar{\psi}\left(a^{\prime}\right) x\right)=f\left(\bar{\psi}\left(y a^{\prime}\right) x\right)=\bar{f}(x)\left(y a^{\prime}\right)=\left(a^{\prime} \bar{f}(x)\right)(y)
$$

where $y \in A\left(g^{\prime}\right)$ is arbitrary. This shows that $\bar{f} \in \operatorname{Hom}_{A\left(g^{\prime}\right)}\left(A(g), \operatorname{Ind}_{{\overline{\mathcal{C}^{\prime \prime}}}_{g^{\prime \prime}}}^{\overline{\mathcal{C}}^{\prime \prime}}(M)\right)$.

In order to prove $\bar{f} \in \operatorname{Ind}_{\overline{\mathcal{C}}_{g^{\prime}}^{\prime}}^{\overline{\mathcal{C}_{g}}} \operatorname{Ind}_{\overline{\mathcal{C}}_{g^{\prime \prime}}^{\overline{\mathcal{C}}^{\prime \prime}}}^{\overline{\mathcal{C}}^{\prime}}(M)$ it is enough to show that $J \bar{f}=0$ where $J \in \mathcal{L}_{g}$ such that $J f=0$. Note that $(J \bar{f})(x)=\bar{f}(x J)=0$ for all $x \in A(g)$ iff $\bar{f}(J)=0$. But $\bar{f}(J)(y)=f(\bar{\psi}(y) J) \subset f(J)=0$ for all $y \in A\left(g^{\prime}\right)$. Thus $\bar{f}(J)=0$.

Next we show that $\xi_{M}$ is a homomorphism of $A(g)$-modules. Let $a \in A(g)$ and $f$, $x, y$ be as before. Then

$$
\xi_{M}(a f)(x)(y)=(a f)(\psi(y) x)=f(x a)(y)=\left(a \xi_{M}(f)\right)(x)(y)
$$

or $\xi_{M}(a f)=a \xi_{M}(f)$.

It remains to show that $\xi_{M}$ is an isomorphism of $A(g)$-modules. We achieve this by constructing an inverse $\eta$ of $\xi$. For $\phi \in \operatorname{Ind}_{\overline{\mathcal{C}}_{g^{\prime}}}^{\bar{C}_{g}} \operatorname{Ind}_{\overline{\mathcal{C}}_{g^{\prime \prime}}}^{\overline{\mathcal{C}}_{g^{\prime}}^{\prime}}(M)$, define $\eta(\phi)=f: A(g) \rightarrow M$ by $f(x)=e v \circ \phi(x)=\phi(x)(1)$ (note that $\phi(x) \in \operatorname{Ind}_{\overline{\mathcal{C}}_{g^{\prime \prime}}^{\prime \prime}}^{\overline{\mathcal{C}}_{g^{\prime}}^{\prime}}(M)$ ). It is easy to verify that $f \in \operatorname{Hom}_{A\left(g^{\prime \prime}\right)}(A(g), M)$. In fact if $a^{\prime \prime} \in A\left(g^{\prime \prime}\right)$ then

$$
\begin{aligned}
f\left(\psi^{\prime \prime}\left(a^{\prime \prime}\right) x\right) & =\phi\left(\psi^{\prime \prime}\left(a^{\prime \prime}\right) x\right)(1)=\left(\psi^{\prime}\left(a^{\prime \prime}\right) \phi(x)\right)(1) \\
& =\phi(x)\left(\psi^{\prime}\left(a^{\prime \prime}\right)\right)=a^{\prime \prime}(\phi(x)(1))=a^{\prime \prime} f(x) .
\end{aligned}
$$


Since there exists $J \in \mathcal{L}_{g}$ such that $J \phi=0$, then

$$
(J f)(x)=f(x J)=\phi(x J)(1)=0 .
$$

Then $f \in \operatorname{Ind}_{\overline{\mathcal{C}}_{g^{\prime \prime}}^{\prime \prime}}^{\overline{\mathcal{C}_{g}}} M$. Thus $\eta_{M}(\phi)=f$ yields a linear map from $\operatorname{Ind}_{\overline{\mathcal{C}}_{g^{\prime}}^{\prime}}^{\overline{\mathcal{C}}_{g}} \operatorname{Ind}_{\overline{\mathcal{C}}_{g^{\prime \prime}}^{\prime \prime}} \overline{\mathcal{C}}^{\prime}(M)$ to $\operatorname{Ind}_{\overline{\mathcal{C}}_{g^{\prime \prime}}}^{\overline{\mathcal{C}}_{g}}(M)$. Moreover, $\eta$ is a homomorphism of $A(g)$-modules:

$$
\eta_{M}(a \phi)(x)=(a \phi)(x)(1)=\phi(x a)(1)=f(x a)=(a f)(x) .
$$

In order to finish the proof we need to check that $\xi_{M} \circ \eta_{M}=i d$ and $\eta_{M} \circ \xi_{M}=i d$. Let $f \in \operatorname{Ind}_{\overline{\mathcal{C}}_{g^{\prime \prime}}^{\prime \prime}}^{\overline{\mathcal{C}}_{g}}(M)$ and $\phi \in \operatorname{Ind}_{\overline{\mathcal{C}}_{g^{\prime}}}^{\overline{\mathcal{C}}_{g}} \operatorname{Ind}_{\overline{\mathcal{C}}_{g^{\prime \prime}}^{g^{\prime \prime}}}^{\overline{\mathcal{C}}^{\prime}}(M)$. Then

$$
\begin{gathered}
\left(\left(\eta_{M} \circ \xi_{M}\right) f\right)(x)=\left(\eta_{M} \bar{f}\right)(x)=\bar{f}(x)(1)=f(x), \\
\left(\left(\xi_{M} \circ \eta_{M}\right) \phi\right)(x)(y)=\left(\overline{\eta_{M} \phi}\right)(x)(y)=\left(\eta_{M} \phi\right)(\bar{\psi}(y) x)=\phi(\bar{\psi}(y) x)(1)=(y \phi(x))(1)=\phi(x)(y)
\end{gathered}
$$
for all $x \in A(g)$ and $y \in A\left(g^{\prime}\right)$. The proof is complete.

3.6. Since $\operatorname{Ind}_{\overline{\mathcal{C}}_{g^{\prime}}^{\prime}}^{\overline{\mathcal{C}}_{g}}$ is left exact and the category $\overline{\mathcal{C}}_{g^{\prime}}^{\prime}$ has enough injective objects we can consider the right derived functors $R^{i} \operatorname{Ind}_{\overline{\mathcal{C}}_{g^{\prime}}}^{\overline{\mathcal{C}}_{g}}$. For each $g^{\prime}$-twisted $V^{\prime}$-module $M$ in $\overline{\mathcal{C}}_{g^{\prime}}^{\prime}, R^{i} \operatorname{Ind}_{\overline{\mathcal{C}}_{g^{\prime}}}^{\overline{\mathcal{C}}_{g}}(M)$ is a $g$-twisted $V$-module in $\overline{\mathcal{C}}_{g^{\prime}}^{\prime}$. Furthermore, since $\operatorname{Ind}_{\overline{\mathcal{C}}_{g^{\prime}}^{\prime}}^{\overline{\mathcal{C}}_{g}}$ sends injective modules to injective modules, we have the Grothendieck spectral sequences with $E_{2}$-terms

$$
R^{i} \operatorname{Ind}_{\overline{\mathcal{C}}_{g^{\prime}}}^{\overline{\mathcal{C}}_{g}} \circ R^{j} \operatorname{Ind}_{{\overline{\mathcal{C}^{\prime}}}_{g^{\prime \prime}}^{\prime \prime}}^{\overline{\mathcal{C}}^{\prime}}(M) \Rightarrow R^{i+j} \operatorname{Ind}_{\overline{\mathcal{C}}_{g^{\prime \prime}}^{\prime \prime}}^{\overline{\mathcal{C}}_{g}}(M)
$$

for each $g^{\prime \prime}$-twisted $V^{\prime \prime}$-module $M$ in $\overline{\mathcal{C}}_{g^{\prime \prime}}^{\prime \prime}$.

3.7. We have already defined the functor $\mathcal{H}$ from the category of vector spaces to $\overline{\mathcal{C}}_{g}$ and proved it to be exact earlier. Now we can see that $\mathcal{H}$ can be defined with the induction functor. Note that our induction even makes sense if we take $A$ being any subalgebra of $A(g)$ such that the corresponding category $\overline{\mathcal{C}}_{A}$ is defined in the following way: an $A$-module $M$ is in $\overline{\mathcal{C}}_{A}$ if and only if $\left\{m \in M \mid(J \cap A) m=0\right.$ for some $\left.J \in \mathcal{L}_{g}\right\}$. In particular we may take $A=\mathbb{C}$. Then $\overline{\mathcal{C}}_{A}=\mathbb{C}$-Vect because for any $J \in \mathcal{L}_{g}$ the intersection $J \cap \mathbb{C}=\{0\}$. Thus

$$
\operatorname{Ind}_{\overline{\mathcal{C}}_{A}}^{\overline{\mathcal{C}}_{g}}(M)=\mathcal{H}(M)
$$




\section{The $g$-rational vertex operator algebras}

4.1. In this section, we consider the induced modules for $g$-rational vertex operator algebras. We call $V g$-rational, if $\mathcal{C}_{g}$ is semisimple with finitely irreducible modules.

However, we still fix an arbitrary vertex operator algebra $V$ and an automorphism $g$ of finite order $K$ of $V$. On each $g$-twisted $V$-module, the operator $L(0)$ acts semi-simply and the graded $A(g)$-module structure is given by the eigenspaces of the operator $L(0)$. Similar to a finite dimensional semisimple Lie algebra, we say $\lambda \leq \mu$ for two weights $\lambda, \mu \in \mathbb{C}$ if $0 \leq \mu-\lambda \in \frac{1}{K} \mathbb{Z}$.

Recall that the algebra $A(g)$ is graded with gradation given by the adjoint action of $L(0)$. The weight space $A(g)_{0}$ is an associate subalgebra of $A(g)$, with the operator $L(0)$ being in the center of $A(g)_{0}$. On every finite dimensional simple $A(g)_{0}$-module, $L(0)$ acts as a scalar.

The subspace $A^{\leq 0}=\oplus_{0 \leq n \in \frac{1}{K} \mathbb{Z}} A(g)_{-n}$ is a graded subalgebra of $A(g)$, and $A^{-}=$ $\oplus_{0<n \in \frac{1}{K} \mathbb{Z}} A(g)_{-n}$ is a graded ideal of $A^{\leq 0}$. Let $M$ be a module in $\mathcal{C}_{g}$, an weight $\lambda$ of $M$ is called a minimal weight if there is no weight of $M$ that is smaller than $\lambda$ in the sense defined above. Note that each weight space $M_{\mu}$ of $M$ is an $A(g)_{0}$-module. However, if $\lambda$ is a minimal weight of $M$, then $M_{\lambda}$ is an $A^{\leq 0}$-module with $A^{-}$acting as zero.

Lemma Let $M$ be an irreducible module in $\mathcal{C}_{g}$, then there is a unique minimal weight $\lambda$ such that $M_{\lambda}$ is a finite dimensional simple $A(g)_{0}$-module.

Proof: By the definition, there exists $\lambda \in \mathbb{C}$ such that $\lambda$ is minimal and $M_{\lambda}$ is not zero. If $\mu$ is a different minimal weight with $M_{\mu} \neq 0$, then $A(g) M_{\mu}=\oplus_{n \in \frac{1}{K} \mathbb{N}} A(g)_{n} M_{\mu} \neq 0$ is a submodule of $M$, which does not contain the subspace $M_{\lambda}$. This contradicts the irreducibility of $M$. Thus $\lambda$ is the only minimal weight. If $0 \neq N \subseteq M_{\lambda}$ is an $A(g)_{0^{-}}$ submodule of $M_{\lambda}$, then $A(g) N=\oplus_{n \in \frac{1}{K} \mathbb{N}} A(g)_{n} N$ is a submodule of $M$ by the Jacobi identity (10), with $p=0$. However we have $(A(g) N)_{\lambda}=N . \quad N \neq 0$ implies that $A(g) N \neq 0$. The irreducibility of $M$ implies that $N=M_{\lambda}$. The finite dimensionality of $M_{\lambda}$ follows from the definition of modules in $\mathcal{C}_{g}$.

A module $M$ in $\mathcal{C}_{g}$ is called a lowest weight module if there is a vector $m \in M_{\lambda}$ for for a minimal weight $\lambda$ such that $M=A(g) m$. Thus all irreducible modules are lowest weight modules by the lemma. For a lowest weight module $M$ of lowest weight $\lambda$, all weights of $M$ are of the form $\lambda+\nu$ with $\nu \in \frac{1}{K} \mathbb{N}$.

4.2. Proposition Let $M^{1}$ and $M^{2}$ be two lowest weight modules $\mathcal{C}_{g}$ with lowest weights $\lambda_{1}$ and $\lambda_{2}$ respectively. If there exists a non-split extension

$$
0 \rightarrow M^{1} \rightarrow E \stackrel{\pi}{\rightarrow} M^{2} \rightarrow 0
$$

in $\mathcal{C}_{g}$, then $\lambda_{1}-\lambda_{2} \in \frac{1}{K} \mathbb{Z}$. 
Proof: Let $m_{2} \in M_{\lambda_{2}}^{2}$ such that $A(g) m_{2}=M^{2}$. Since $E$ is in $\mathcal{C}_{g}$, there exists $0 \neq m \in$ $E_{\lambda_{2}}$ such that $\pi(m)=m_{2}$. Then we consider the submodule $A(g) m$ of $E$. All weights of $A(g) m$ are in $\lambda_{2}+\frac{1}{K} \mathbb{Z}$. If $A(g) m \cap M^{1}=0$ then $\pi: A(g) m \rightarrow M^{2}$ is an isomorphism since $\pi(A(g) m)=M^{2}$. This contradicts the nonsplitness of the exact sequence. Thus we must have $A(g) m \cap M^{1} \neq 0$ which is a $g$-twisted $V$-submodule of $M^{1}$. We pick $0 \neq x \in A(g) m \cap M^{1}$. We can assume that $x$ is a weight vector of weight $\nu$. Thus $\nu \in \lambda_{1}+\frac{1}{K} \mathbb{Z}$. But we also have $\nu \in \lambda_{2}+\frac{1}{K} \mathbb{Z}$. This shows that $\lambda_{1}-\lambda_{2} \in \frac{1}{K} \mathbb{Z}$.

4.3. Let $N$ be a finite dimensional $A(g)_{0}$-module such that $L(0)$ acts on it by a scalar $\lambda$. Then we can extend $N$ to an $A^{\leq 0}$-module by letting $A^{-}$act as zero. We consider the $A(g)$-module $M(N)=A(g) \otimes_{A \leq 0} N$. However, $M(N)$ needs not be in $\overline{\mathcal{C}_{g}}$. The followings are some standard properties of the module $M(N)$ :

(1) $M(N)$ is a graded $A(g)$-module with the gradation given by the eigenspaces of the operator $L(0)$ and the projection $\mathrm{pr}_{\lambda}: M(N)_{\lambda} \rightarrow N$ is an isomorphism of the vector spaces (actually as $A^{\leq 0}$-modules).

(2) All weights of $M(N)$ are in $\lambda+\frac{1}{K} \mathbb{N}$.

(3) Suppose that $N$ is a simple $A(g)_{0}$-module. Then there is a nonzero quotient of $M(N)$ in the category $\mathcal{C}_{g}$ if and only if there is a simple module in the category $\mathcal{C}_{g}$ with lowest weight space isomorphic to $N$ as $A(g)_{0}$-modules. Furthermore, if $M(N)$ has any nonzero quotient in $\mathcal{C}_{g}$, then it has a unique maximal submodule and thus a unique simple quotient in $\mathcal{C}_{g}$. Here any $A(g)$-submodule containing a weight vector of the lowest weight $\lambda$ contains the whole module $M(N)$ since $N$ is simple while the sum of all submodules not containing the weight $\lambda$ is still such a submodule.

(4) Sharpiro's lemma holds: for any module $M$ in $\overline{\mathcal{C}}_{g}$ and any finite dimensional $A(g)_{0}$-module $N$, we have the canonical isomorphism

$$
\operatorname{Hom}_{A(g)}(M(N), M)=\operatorname{Hom}_{A(g)_{0}}\left(N, M_{\lambda}^{A^{-}}\right) .
$$

Here $M_{\lambda}^{A^{-}}$is the subspace of $M_{\lambda}$ consisting all vectors killed by all elements of $A^{-}$.

Remark. It is not known in general the $A(g)$-module $M(N)$ has a unique maximal quotient in $\overline{\mathcal{C}}_{g}$, which we would like to denote by $D(N)$. However we expect that this is the case. If the module $D(N)$ exists and not zero, then in the above listed properties we can simply replace $M(N)$ by $D(N)$ so that all the above listed properties hold when $M(N)$ is replaced by $D(N)$ except the isomorphism of the projection in (1).

4.4. Proposition Let $M^{1}$ and $M^{2}$ be two irreducible modules in $\mathcal{C}_{g}$ with lowest weights $\lambda_{1}$ and $\lambda_{2}$ respectively. Then $M^{1}$ and $M^{2}$ are isomorphic in $\mathcal{C}_{g}$ if and only if $M_{\lambda_{1}}$ and $M_{\lambda_{2}}$ are isomorphic as $A(g)_{0}$-modules.

Proof: One direction of the proposition is clear. We only show that $M^{1}$ and $M^{2}$ are isomorphic if $M_{\lambda_{1}}$ and $M_{\lambda_{2}}$ are isomorphic as $A(g)_{0}$-modules. We first observe that 
that $\lambda_{1}=\lambda_{2}$ (we denote them by $\lambda$ ) since they are the scalars by which the operator $L(0)$ acts on the two modules respectively. Let $N^{1}=M_{\lambda}^{1}$ and $N^{2}=M_{\lambda}^{2}$. Then $N^{1}$ and $N^{2}$ are irreducible $A(g)_{0}$. We consider the $A(g)$-module $M\left(N^{1}\right)$. By (4) of the previous subsection, we have

$$
\operatorname{Hom}_{A(g)}\left(M\left(N^{1}\right), M^{2}\right)=\operatorname{Hom}_{A(g)_{0}}\left(N^{1}, N^{2}\right) \neq 0 .
$$

Thus $M\left(N^{1}\right)$ has a unique simple quotient, which has to be isomorphic to $M^{2}$ since $M^{2}$ is simple. We can replace $M^{2}$ by $M^{1}$ in the above argument to show that $M^{1}$ is also a simple quotient of $M\left(N^{1}\right)$. Thus $M^{1} \cong M^{2}$ by the uniqueness of the simple quotient of $M\left(N^{1}\right)$.

Remark. This proposition is related to some results obtained in [DLM] (also see [Z]). In $[\mathrm{DLM}]$ an associative algebra $A_{g}(V)$ associated with the vertex operator algebra $V$ and the automorphism $g$ is constructed. It is proved there that there is a bijection between the set of inequivalent weak irreducible $g$-twisted modules (the eigenspace of $L(0)$ in a weak module can be infinite-dimensional) and the set of inequivalent irreducible $A_{g}(V)$-modules. One can easily verify that $A_{g}(V)$ is a quotient of $A(g)_{0}$.

4.5. Recall that a block $\mathcal{B}$ in $\mathcal{C}_{g}$ is a full subcategory of $\mathcal{C}_{g}$ generated by an equivalent class of irreducible modules with respect to the equivalent relation generated by the following: $M^{1}$ and $M^{2}$ are equivalent if there is a non-split extension in $\mathcal{C}_{g}$ between them. By Lemma 4.2, each block uniquely determines an element in $\mathbb{C} /\left(\frac{1}{K} \mathbb{Z}\right)$ by taking the images of the lowest weights of the irreducible modules in the block. We say that a block is bounded above if $\lambda+\frac{1}{K} \mathbb{Z}$ is the class in $\mathbb{C} /\left(\frac{1}{K} \mathbb{Z}\right)$ corresponding to the block $\mathcal{B}$ and the set of lowest weights of all irreducible modules in $\mathcal{B}$ is a subset of $\lambda+\frac{1}{K} \mathbb{Z}$ with an upper bound.

Proposition Suppose that all blocks in $\mathcal{C}_{g}$ are bounded above, then every $A(g)$ finitely generated module in $\mathcal{C}_{g}$ has a composition series.

Proof: Let $M$ be a finitely generated module in $\mathcal{C}_{g}$. Without lose of generality, we can assume that $M$ is generated by finitely many weight vectors, say, $m_{1}, \ldots, m_{n}$ with weights $\lambda_{1}, \ldots, \lambda_{n}$. Consider the finite filtration of submodules generated by these vectors:

$$
0=M^{0} \subseteq M^{1}=\left\langle m_{1}\right\rangle \subseteq\left\langle m_{1}, m_{2}\right\rangle \subseteq \cdots \subseteq M^{n}=\left\langle m_{1}, \ldots, m_{t}\right\rangle=M .
$$

We see that each subquotient $M^{i} / M^{i-1}$ is generated by a single weight vector. Thus we can assume that $M$ is generated by a single vector. Thus all weights of $M$ are of the form $\lambda+\frac{1}{K} \mathbb{Z}$. Furthermore since all the weight spaces are finite dimensional and only finitely many weight spaces of weights $\lambda-\frac{j}{K}$ with $j \in \mathbb{N}$. By a similar argument, we can assume that the generator is a lowest weight vector. Thus $M$ is a lowest weight module. 
Now we need to use the condition that every block is bounded above. Let $n$ be the positive integer such that there is no irreducible module of lowest weights of the form $\lambda+\frac{j}{K}$ with $j>n$. We can choose $n=n_{\lambda}$ as the smallest for the each such fixed $\lambda$ and use induction on $n_{\lambda}$. If $n_{\lambda}=0$, we note that $M_{\lambda}$ is finite dimensional. Take $N \subseteq M_{\lambda}$ as an irreducible $A(g)_{0}$-submodule. Consider the $A(g)$-module $M(N)$. We have a nonzero homomorphism $\phi: M(N) \rightarrow M$ resulted from the embedding $N \rightarrow M_{\lambda}$. Then $M(N)$ has a nonzero quotient in $\mathcal{C}_{g}$ and the unique maximal submodule $M(N)_{m}$ has all weights of the form $\lambda+\frac{j}{K}$ with $j>0$. If the image of $\phi$ is not irreducible, it has to have a submodule with all weights of the form $\lambda+\frac{j}{K}$ with $j>0$. It has to have a lowest weight vector which generates a submodule with a simple quotient in $\mathcal{C}_{g}$ with lowest weight larger than $\lambda$. This contradicts the assumption that $n_{\lambda}=0$. Now we can use a induction on the dimension of $M_{\lambda}$ and reduce the proof to the case such that $M_{\lambda}$ is irreducible as $A(g)_{0}$-module. A similar argument shows that $M$ has to be simple in this case. For $n_{\lambda}>0$, We can use a similar argument to find submodules $F \subseteq E$ of $M$ such that $E / F$ is irreducible with lowest weight $\lambda$ and both $F$ and $M / E$ have either larger lowest weight or same lowest weight but strictly smaller dimension of the lowest weight space. By induction we see that both $F$ and $M / E$ have composition series. Thus $M$ has a composition series.

Corollary With the conditions in as in the Theorem, all modules in $\mathcal{C}_{g}$ with weights in a finite union $\cup_{i=1}^{n}\left(\lambda_{i}+\frac{\mathbb{Z}}{K}\right)$ have composition series.

Actually the proof of the Theorem works for such assumption.

4.6. Proposition If all blocks in $\mathcal{C}_{g}$ are bounded above, then any non-zero module in $\mathcal{C}_{g}$ has non-zero simple submodules and non-zero simple quotient.

Proof: Consider for each $\lambda \in \mathbb{C}$ the subspace $M(\lambda)=\sum_{i \in \mathbb{Z}} M_{\lambda+\frac{i}{K}}$ which, in fact, is a submodule of $M$. By Lemma 4.2, we must have $M=\oplus_{\lambda \in \mathbb{C} / \frac{z}{K}} M(\lambda)$. By Corollary 4.5, each $M(\lambda)$ has a composition series if it is not zero. Thus each non-zero $M(\lambda)$ has a non-zero simple submodule and quotient.

4.7. Lemma Let $V^{\prime}$ be a $g$-invariant vertex subalgebra of $V$ and $g^{\prime}=\left.g\right|_{V^{\prime}}$. If $\mathcal{C}_{g^{\prime}}^{\prime}$ is bounded above, so is $\mathcal{C}_{g}$.

Proof: Let $M$ be a simple module in $\mathcal{C}_{g}$. Then $M$ is a module in $\mathcal{C}_{g^{\prime}}^{\prime}$. The lowest weight space $M_{\lambda}$ generates a submodule in $\mathcal{C}_{g^{\prime}}^{\prime}$, which has a simple subquotient of lowest weight $\lambda$. That the $\mathcal{C}_{g^{\prime}}^{\prime}$ is bounded above implies that $\mathcal{C}_{g}$ is necessarily bounded above.

4.8. A natural question about the induction functor is when $\operatorname{Ind}_{\overline{\mathcal{C}}_{g^{\prime}}^{\prime}}^{\overline{\mathcal{C}}_{g}} N$ is not zero for an irreducible module $N$ in $\mathcal{C}_{g^{\prime}}^{\prime}$. Since we are interested in modules in $\mathcal{C}_{g}$ only. So 
another natural question is whether the module $\operatorname{Ind}_{\overline{\mathcal{C}}_{g^{\prime}}^{\prime}}^{\overline{\mathcal{C}}_{g}} N$ is in $\mathcal{C}_{g}$ whenever $N$ is in $\mathcal{C}_{g^{\prime}}^{\prime}$. We discuss these questions in the following special case. We start with the following lemma.

Lemma Let $L$ and $M$ be modules in $\mathcal{C}_{g}$ such that $L$ is finitely generated. Then $\operatorname{Hom}_{\mathcal{C}_{g}}(L, M)$ is finite dimensional.

Proof: Since $L$ is finitely generated, we can further assume that the finite set of generators are all weight vectors. Let $N$ be the subspace generated by the generators, which is a finite direct sum of finite dimensional weight spaces. Let $\phi: L \rightarrow M$ be any homomorphism in $\mathcal{C}_{g}$. Then $\phi$ is uniquely determined by the restriction map $\phi_{0}$ : $N \rightarrow M_{0}$. Here $M_{0}$ is the sum of the weight subspaces of $M$ corresponding to all the weights that appear in $N$. Now both $N$ and $M_{0}$ are finite dimensional.

Remark. A similar argument shows that if $M$ has a composition series, then $\operatorname{Hom}_{\mathcal{C}_{g}}(L, M)$ is finite dimensional for all $L$ in $\mathcal{C}_{g}$. This can be reduced to the case that $M$ is irreducible. Then one can use the fact that any module in $\mathcal{C}_{g}$ can not have a quotient which is a direct sum of an irreducible module infinitely many times due to the finite dimensionality of the weight spaces of modules in $\mathcal{C}_{g}$.

Theorem Let $V$ be a g-rational vertex operator algebra. Then $\operatorname{Ind}_{{\overline{\mathcal{C}^{\prime}}}_{g^{\prime}}}^{\overline{\mathcal{C}}_{g}} L$ is in $\mathcal{C}_{g}$ for every irreducible module $L$ in $\mathcal{C}_{g^{\prime}}^{\prime}$. More generally, $\operatorname{Ind}_{\overline{\mathcal{C}}_{g^{\prime}}^{\prime}}^{\overline{\mathcal{C}}_{g}} N$ is in $\mathcal{C}_{g}$ for any module $N$ in $\mathcal{C}_{g^{\prime}}^{\prime}$ which has a composition series.

Proof: First of all, since $\mathcal{C}_{g}$ has only finitely many irreducible modules, then any module in $\mathcal{C}_{g}$ has a composition series. Thus a module in $\overline{\mathcal{C}}_{g}$ is non-zero if and only if it has a non-zero simple submodule. Take any simple module $S$ in $\mathcal{C}_{g}$. By the Frobenius reciprocity, we have $\operatorname{Hom}_{\overline{\mathcal{C}}_{g}}\left(S, \operatorname{Ind}_{\overline{\mathcal{C}}_{g^{\prime}}}^{\overline{\mathcal{C}}_{g}} L\right)=\operatorname{Hom}_{\mathcal{C}_{g^{\prime}}^{\prime}}(S, L)$. Since $L$ is simple in $\mathcal{C}_{g^{\prime}}^{\prime}, \operatorname{Hom}_{\mathcal{C}_{g^{\prime}}^{\prime}}(S, L)$ is finite dimensional following the above remark. Thus $S$ appears in $\operatorname{Ind}_{\overline{\mathcal{C}}_{g^{\prime}}^{\prime}}^{\overline{\mathcal{C}}_{g}} L$ only finitely many times as direct summand since the category $\mathcal{C}_{g}$ is semisimple (thus $\overline{\mathcal{C}}_{g}$ is also semisimple). Since $\mathcal{C}_{g}$ has only finitely many irreducible modules, $\operatorname{Ind}_{\overline{\mathcal{C}}_{g^{\prime}}}^{\overline{\mathcal{C}}_{g}} L$ is a direct sum of finitely many irreducible modules and thus in $\mathcal{C}$.

\section{Inductions for simple vertex operator algebras}

5.1. In this section we discuss the case when $V$ is a simple vertex operator algebra. Fix an automorphism $g$ of finite order $K$ of $V$. Then $V=\oplus_{r=0}^{K-1} V^{r}$ is a direct sum decomposition of $g$-eigenspaces. It is proved in [DM1] that $V^{\prime}=V^{0}$ is a simple vertex 
operator algebra and $V^{r}$ is an irreducible $V^{0}$-module. Note that for any $r, u \in V^{r}$ homogeneous, and any $g$-twisted module $M$ in $\mathcal{C}_{g}$, the degree of the operator $u_{n}$ (as a linear operator on the graded vector space $M)$ equals wt $(u)-n-1$ with $n \in \frac{r}{K}+\mathbb{Z}$. In particular, for each $u \in V^{0}, u_{n}$ has degree in $\mathbb{Z}$. Thus for any $g$-twisted $V$-module $M$ and $\lambda \in \mathbb{C}$ the vector space $M^{0}(\lambda)=\oplus_{n \in \mathbb{Z}} M_{\lambda+n}$ is a $V^{0}$-submodule of $M$. Note that $M^{0}(\lambda)=M^{0}(\mu)$ if and only if $\lambda-\mu \in \mathbb{Z}$. On the other hand, $M(\lambda)=\oplus_{n \in \frac{1}{K} \mathbb{Z}} M_{\lambda+n}$ is an $g$-twisted $V$-submodule of $M$ and $M(\lambda)=M(\mu)$ if and only if $\lambda-\mu \in \frac{1}{K} \mathbb{Z}$. Therefore, we have $M(\lambda)=\oplus_{r=0}^{K-1} M^{0}\left(\lambda+\frac{r}{K}\right)$, which gives a decomposition as $V^{0}$-modules.

For each fixed $\lambda$, we can define a $\langle g\rangle$-action on $M(\lambda)$ such that $g$ acts on $M^{0}\left(\lambda-\frac{r}{K}\right)$ by the scalar $\xi^{r}$. Here we fix $\xi=e^{\frac{2 \pi i}{K}}$.

Lemma With the above action, we have

$$
g \circ Y_{M(\lambda)}(u, z) \circ g^{-1}=Y_{M(\lambda)}(g u, z)
$$

for all $u \in V$.

Proof: Let $u \in V^{r}$ and $m \in M^{0}\left(\lambda+\frac{j}{K}\right)$ be homogeneous elements. For each $n \in \frac{r}{K}+\mathbb{Z}$, $u_{n}(m)$ has weight wt $v+$ wt $m-n-1 \in$ wt $m-\frac{r}{K}+\mathbb{Z}$. Thus $u_{n}(m) \in M^{0}\left(\lambda-\frac{j}{K}-\frac{r}{K}\right)$ and

$$
g\left(u_{n}\left(g^{-1} m\right)\right)=g\left(\xi^{-j} u_{n}(m)\right)=\xi^{-j} \xi^{j+r} u_{n}(m)=\xi^{r} u_{n}(m)=\left(\xi^{r} u\right)_{n} m=(g u)_{n} m .
$$

Remark. Through the decomposition $M=\oplus_{\lambda \in \mathbb{C} /\left(\frac{\mathrm{Z}}{K}\right)} M(\lambda), g$ acts on $M$. However, this action is not functorial and depends on the choice of the $\lambda$ in $\lambda+\frac{\mathbb{Z}}{K}$.

5.2. Let $h$ be an automorphism of $V$, commuting with $g$. Then $h$ preserves the $g$-eigenspace decomposition of $V$. Let $M$ be any $g$-twisted module with the vertex operators denoted by $Y_{M}(v, z)$. Let ${ }^{h} M=M$ as vector spaces. For each $v \in V$, we define $Y_{{ }} M(v, z)=Y_{M}(h v, z)$. It is straightforward to verify that $Y_{{ }^{h}} M(v, z)$ makes ${ }^{h} M$ into a $g$-twisted $V$-module. If $\phi: M \rightarrow N$ is a homomorphism of $g$-twisted $V$-modules, then ${ }^{h} \phi=\phi:{ }^{h} M \rightarrow^{h} N$ is also a homomorphism of $g$-twisted $V$-modules. Thus the assignment $M \mapsto{ }^{h} M$ defines a functor $\mathcal{C}_{g} \rightarrow \mathcal{C}_{g}$ and ${ }^{\left(h_{1} h_{2}\right)} M={ }^{h_{1}}\left({ }^{h_{2}} M\right)$.

Lemma Let $M$ be a $g$-twisted $V$-module, then for any choice of the action $g: M \rightarrow$ $M$ defined in the remark above gives an isomorphism $M \rightarrow{ }^{h} M$ of $g$-twisted modules.

Proof: For each $\lambda$, the action of $g$ on $M(\lambda)$ defines an isomorphism of $g$-twisted $V$ modules by Lemma 5.1. Then the isomorphism extends to $M$ through the direct sum decomposition. 
5.3. Recall that for each homogeneous $u \in V^{r}$, the weight of $u_{n}$ is in $\mathbb{Z}-\frac{r}{K}$ as operator on any $g$-twisted $V$-modules. We set $A^{r}=\oplus_{n \in \mathbb{Z}} A(g)_{n-\frac{r}{K}}$. Then we have

$$
A(g)=\oplus_{r=0}^{K-1} A^{r}
$$

which gives another graded algebra structure on $A(g)$.

Since for each automorphism $h$ of $V$ commuting with $g$, the module ${ }^{h} M$ is also a $g$ twisted module, one verifies directly from the definition that $h\left(u_{n}\right)=(h u)_{n}$ defines an automorphism of the associated algebra $A(g)$. In particular $g$ defines an automorphism of $A(g)$ such that the gradation $A(g)=\oplus_{r=0}^{K-1} A^{r}$ is given by the $g$-eigenspaces with $g$ acts on $A^{r}$ by $\xi^{r}$.

Now let $M$ be any module in $\mathcal{C}_{g}$. The decomposition $M(\lambda)=\oplus_{r=0}^{K-1} M^{0}\left(\lambda-\frac{r}{K}\right)$ is a graded $A(g)$-module with respect to the above gradation of $A(g)$. This graded $A(g)$ module structure is independent of the choice of $\lambda$ up to the index shifting. Moreover, for any fixed $g$ action on $M$ compatible with the $g$-action $V$ in the sense of Lemma 5.1 , the $g$-eigenspace decomposition of $M$ gives the graded $A(g)$-module structure on $M$.

We will concentrate on the case when $M$ is simple. If we assume the lowest weight spaces is contained in the $g$-invariant spaces of $M$, then there is a unique way to index the homogeneous spaces such that the lowest weight space is in $M^{0}$ and we will denote them by $M=M^{0} \oplus M^{1} \oplus \cdots \oplus M^{K-1}$. This notation is compatible to the case when $V$ is a simple $V$-module (here $g=1$ ).

Our next goal is to show that if $M$ simple, then $M^{0}, M^{1}, M^{K-1}$ are non-isomorphic simple (untwisted) $V^{0}$-modules. In the following $V$ is still an arbitrary vertex operator algebra. The result is a strong contrast with Lie algebra and indicates the associative algebra feature of vertex operator algebras.

5.4. Proposition Let $M$ be a module in $\overline{\mathcal{C}}_{g}$ and $m \in M$ be homogeneous. Then the subspace $\operatorname{Ann}_{V}(m)=\left\{v \in V \mid Y_{M}(v, z) m=0\right\}$ is a $V$-submodule of $V$ (thus an ideal of $V$ ).

Proof: Since $m$ generates a submodule of $M$ in $\mathcal{C}_{g}$. We can assume that $M$ is in $\mathcal{C}_{g}$. Thus all weight spaces are finite dimensional. For any $v \in V$, we can write $v=$ $v^{0}+\cdots+v^{K-1}$ such that $v^{r} \in V^{r}$. By considering the weights, we have $Y_{M}(v, z) m=0$ if and only if $Y_{M}\left(v^{r}, z\right) m=0$ for all $r$. Thus $\operatorname{Ann}_{V}(m)=\oplus_{r=0}^{K-1}\left(V^{r} \cap \operatorname{Ann}_{V}(m)\right)$.

Let $M^{\prime}=\oplus_{\lambda} M_{\lambda}^{*}$ be the graded dual of $M$. We need to discuss the associativity of the vertex operator product expansion (cf. [DM2], [DL]). Let $\mathbb{C}\left[z_{1}, z_{2}\right]_{S}$ be the localization of the polynomial ring $\mathbb{C}\left[z_{1}, z_{2}\right]$ with respect to the set of all nonzero homogeneous polynomials of degree 1 and $\iota_{i_{1} i_{2}}: \mathbb{C}\left[z_{1}, z_{2}\right]_{S} \rightarrow \mathbb{C}\left[\left[z_{1}, z_{1}^{-1}, z_{2}, z_{2}^{-1}\right]\right]$ the injective map such that an element $\left(a z_{1}+b z_{2}\right)^{-1}$ is expanded in nonnegative integral powers of $z_{i_{2}}$ where $\left(i_{1}, i_{2}\right)$ is an ordering of the set $\{1,2\}$. Let $v \in V^{i} \cap \operatorname{Ann}_{V}(m)$ and $u \in V^{j}$. For 
each $m^{\prime} \in M^{\prime}$ by the rationality for $g$-twisted modules, there exists $f\left(z_{1}, z_{2}\right) \in \mathbb{C}\left[z_{1}, z_{2}\right]_{S}$ such that

$$
m^{\prime}\left(Y_{M}\left(u, z_{1}\right) Y_{M}\left(v, z_{2}\right) m\right) z_{1}^{\frac{j}{K}} z_{2}^{\frac{i}{K}}=\iota_{12} f\left(z_{1}, z_{2}\right)
$$

and by the associatively, we have

$$
m^{\prime}\left(Y_{M}\left(Y\left(u, z_{0}\right) v, z_{2}\right) m\right)\left(z_{0}+z_{2}\right)^{\frac{j}{K}} z_{2}^{\frac{i}{K}}=\iota_{20} f\left(z_{0}+z_{2}, z_{2}\right)
$$

for the same $f$. Since $v \in \operatorname{Ann}_{V}(m)$, we have $\iota_{12} f\left(z_{1}, z_{2}\right)=0$, which implies $f\left(z_{1}, z_{2}\right)=$ 0 . Thus $f\left(z_{0}+z_{2}, z_{2}\right)=0$ and $\iota_{20} f\left(z_{0}+z_{2}, z_{2}\right)=0$. Therefore

$$
m^{\prime}\left(Y_{M}\left(Y\left(u, z_{0}\right) v, z_{2}\right) m\right)=0
$$

Since $m^{\prime} \in M^{\prime}$ is arbitrary, we have $Y_{M}\left(Y\left(u, z_{0}\right) v, z_{2}\right) m=0$. Thus $u_{n}(v) \in \operatorname{Ann}_{V}(m)$ and $\operatorname{Ann}_{V}(m)$ is a $V$-submodule of $V$.

Corollary Suppose $V$ is a simple vertex operator algebra. Let $M$ be a module in $\mathcal{C}_{g}$ with any fixed $g$-action on $M$ such the resulted eigenspace decomposition $M=$ $\oplus_{r=0}^{K-1} M^{r}$ gives a graded $A(g)$-module as in5.3. Then $M \neq 0$ implies $M^{r} \neq 0$.

Proof: We may assume that $M$ is irreducible and the gradation is the canonical one. Fix a nonzero vector $m$ in the lowest weight space. Then we have $M=A(g) m=$ $\oplus_{r=0}^{K-1} A^{r} m$ and $A^{r} m \subseteq M^{r}$. Thus we have the equality $A^{r} m=M^{r}$. Note that $u_{n} \in A^{r}$ for all $n \in \frac{1}{K} \mathbb{Z}$ whenever $u \in V^{r}$. Suppose $M^{r}=0$ for some $r$. Then we have $V^{r} \subseteq \operatorname{Ann}_{V}(m)$. Thus the submodule $\operatorname{Ann}_{V}(m) \neq 0$ by Theorem 2 of [DM1]. However, 1 is not in $\operatorname{Ann}_{V}(m)$. This contradicts the simplicity of $V$.

5.5. Recall from 2.3 that $\bar{V}=\oplus_{i=0}^{k-1}\left(V^{i} \otimes t^{i / K} \mathbb{C}\left[t, t^{-1}\right]\right)$ and there is a natural homomorphism $\bar{V}$ to $A(g)$. Thus $\bar{V}$ "acts" on every module in $\mathcal{C}_{g}$. Note that $\bar{V}$ is only a subspace in $A(g)$. However the following explains that $U_{g}(V)$ is not much larger than $\bar{V}$. The proof of the lemma uses a similar argument as in [DM1] for the ordinary module cases by using the rationality and associativity properties for $g$-twisted modules. For simplicity we denote $\bar{V}^{r}=V^{r} \otimes t^{\frac{r}{K}} \mathbb{C}\left[t, t^{-1}\right]$. Then $\bar{V}=\oplus_{r=0}^{K-1} \bar{V}^{r}$.

Lemma Let $V$ be any vertex operator algebra and $g$ is an automorphism of finite order $K$ of $V$. If $M$ is a $g$-twisted $V$-module generated by a set $S$ over $V$, i.e., $M=$ $U_{g}(V) S$, then $M$ is the linear span of the following subset $\left\{u_{n} s \mid u \in V, n \in \frac{\mathbb{Z}}{K}, s \in S\right\}$, or simply $M=\bar{V} S$.

Theorem Let $V$ be a simple vortex operator algebra and $M$ a simple $g$-twisted module. Then, in the decomposition $M=M^{0}+M^{1}+\cdots M^{K-1}, M^{0}, \ldots, M^{K-1}$ are nonzero and non-isomorphic simple $V^{0}$-modules. 
Proof: By the corollary above, we know that $M^{i}$ s are nonzero. Next we show that they are irreducible. Let $0 \neq m \in M^{i}$. Since $M$ is simple, thus $S=\{m\}$ is a generating set of $M$ on $V$. By the lemma above, we have $M=\bar{V}=\oplus_{r=0}^{K-1} \bar{V}^{r} S$. However, $\bar{V}^{r} S \subseteq M^{i-r}$. This implies that we have the equality $M^{i-r}=\bar{V}^{r} S$. In particular, we have $\bar{V}^{0} S=M^{i}$ and $m$ generates the entire module $M^{i}$ over $V^{0}$. This shows the simplicity of $M^{i}$.

To show that $M^{0}, \ldots, M^{K-1}$ are non-isomorphic $V^{0}$-modules, one can follow a similar argument as in the proof of Theorem 4.1 of [DM1].

5.6. Theorem Let $V$ be a simple vertex operator algebra and $g$ is an automorphism of order $K$. Suppose $M$ and $N$ are two irreducible modules in $\mathcal{C}_{g}$ such that a $V^{0}$-component $M^{r}$ of $M$ is isomorphic to a $V^{0}$-component $N^{s}$ of $N$. Then $M \cong N$ in $\mathcal{C}_{g}$.

Proof: Let $M=M^{0}+\cdots+M^{K-1}$ and $N=N^{0}+\cdots+N^{K-1}$ be $V^{0}$-decompositions of $M$ and $N$ respectively. Suppose $\phi: M^{r} \rightarrow N^{s}$ is an isomorphism of $V^{0}$-modules. Then $\psi: M^{r} \rightarrow M^{r} \oplus N^{s}$ defined by $\psi(m)=(m, \phi(m))$ is a $V^{0}$-module homomorphism with image isomorphic to $M^{r}$ and a proper submodule of $M^{r} \oplus N^{s}$. We now fix $0 \neq m \in M^{r}$. Consider the $g$-twisted $V$-submodule $W$ generated by $(m, \phi(m))$ in $M \oplus N$. Then we have $W=\bar{V}(m, \phi(m))=\sum_{i=0}^{K-1} \bar{V}^{i}(m, \phi(m))$. However, $\bar{V}^{i}(m, \phi(m)) \subseteq M^{r-i} \oplus N^{s-i} \subseteq$ $M \oplus N$ for all $i$. In particular for $i=0$, we have $\bar{V}^{0}(m, \phi(m))=\psi\left(M^{r}\right)$ is a proper submodule of $M^{r} \oplus N^{s}$. Therefore, $W$ is a proper submodule of $M \oplus N$ and $W$ must be a simple submodule. Since both projections $\mathrm{pr}_{1}: W \rightarrow M$ and $\mathrm{pr}_{2}: W \rightarrow N$ are nonzero, they must be isomorphisms, which gives an isomorphism between $M$ and $N$ in $\mathcal{C}_{g}$.

5.7. Now we consider the induction from $V^{0}$ to $V$ for a simple vertex operator algebra $V$ with an automorphism $g$ of finite order. Let $\mathcal{C}_{1}^{0}$ be the category of untwisted $V^{0}$-modules.

Proposition Let $M$ be any simple module in $\mathcal{C}_{g}$. Then $\operatorname{Ind}_{\overline{\mathcal{C}}_{1}^{0}}^{\overline{\mathcal{C}}_{g}} M^{r} \neq 0$. More prescisely, $\operatorname{Ind}_{\overline{\mathcal{C}}_{1}^{0}}^{\overline{\mathcal{C}}_{g}} M^{r}$ has a unique simple submodule isomorphic to $M$.

Proof: Note that the projection $M \rightarrow M^{r}$ is nonzero homomorphism of $V^{0}$-modules. By the Frobenius reciprocity we have a non-zero homomorphism $M \rightarrow \operatorname{Ind}_{\overline{\mathcal{C}}_{1}^{0}}^{\overline{\mathcal{C}}_{g}} M^{r}$. Since $M$ is irreducible in $\mathcal{C}_{g}, M$ is isomorphic to a submodule of $\operatorname{Ind}_{\overline{\mathcal{C}}_{1}^{0}}^{\bar{c}_{g}} M^{r}$. By Theorem 5.5, we have $\operatorname{Hom}_{\mathcal{C}_{1}^{0}}\left(N, M^{r}\right)=\mathbb{C}$. This shows that $M$ appears in the socle of $\operatorname{Ind}_{\overline{\mathcal{C}}_{1}^{0}}^{\overline{\mathcal{C}}_{g}} M^{r}$ exactly once as a direct summand. On the other hand for any simple module $N$ in $\mathcal{C}_{g}$, $\operatorname{Hom}_{\mathcal{C}_{1}^{0}}\left(N, M^{r}\right) \neq 0$ if and only if $N \cong M$ by Theorem 5.6. 
5.8. Theorem Let $V$ be a simple vertex operator algebra. Suppose that $\mathcal{C}_{g}$ is semisimple.

(1) Then for each simple $V^{0}$-module $N$,

$$
\operatorname{Ind}_{\overline{\mathcal{C}}_{1}^{0}}^{\overline{\mathcal{C}}_{g}} N \cong\left\{\begin{aligned}
M & \text { if } N \cong M^{r} \text { for a simple module } M \text { in } \mathcal{C}_{g} \text { and some } r \\
0 & \text { otherwise }
\end{aligned}\right.
$$

(2) If we further assume that $\mathcal{C}_{g}$ has only finitely many simple modules, then for any module $N$ in $\mathcal{C}_{1}^{0}, \operatorname{Ind}_{\mathcal{C}_{1}^{0}}^{\overline{\mathcal{C}}_{g}} N$ is in $\mathcal{C}_{g}$.

Proof: By applying the Frobenius reciprocity and using the Theorem 5.5, we see that the identity of (1) holds if the left hand side is replaced by its socle. Since $\mathcal{C}_{g}$ is semisimple, then $\operatorname{Ind}_{\overline{\mathcal{C}}_{1}^{0}}^{\overline{\overline{\mathcal{C}}_{g}}} N$ is also necessarily semisimple by Proposition 2.9. Thus (1) is proved.

For the same reason as above, $\operatorname{Ind}_{\overline{\mathcal{C}}_{1}^{0}}^{\overline{\mathcal{C}}_{g}} N$ is semisimple. Since for each simple module in $\mathcal{C}_{g}$, it has only finitely many irreducible direct summands as a $V^{0}$-module. On the other hand, for each simple module $L$ in $\mathcal{C}_{g}, \operatorname{Hom}_{\mathcal{C}_{1}^{0}}(L, N)$ is finite dimensional by Lemma 4.8. Therefore $\operatorname{Hom}_{\mathcal{C}_{1}^{0}}(M, N)$ is finite dimensional as well for any simple $g$ twisted $V$-module $M$ by Theorem 5.5. Thus by the Frobenius reciprocity, each simple $g$-twisted $V$-module appears only finitely many times as a direct summand of $\operatorname{Ind}_{\overline{\mathcal{C}}_{1}^{0}}^{\overline{\mathcal{C}}_{g}} N$. Since $\mathcal{C}_{g}$ has only finitely many simple modules. Then $\operatorname{Ind}_{\overline{\mathcal{C}}_{1}^{0}}^{\bar{c}_{g}} N$ is a direct sum of finitely simple modules and thus in $\mathcal{C}_{g}$.

Remark. If $V$ is simple and rational (i.e., $g$-rational with $g=1$ ), the above theorem implies that $V=\operatorname{Ind}_{\mathcal{C}_{1}^{0}}^{\overline{\mathcal{C}}_{1}} V^{0}$ if we consider the ordinary modules. The induction functor is actually defined from $\mathcal{C}_{1}^{0}$ to $\mathcal{C}_{g}$.

\section{References}

[APW] H.H. Andersen, P. Polo, K. Wen, Representations of quantum algebras, Invent. Math. 104 (1991), 1-59.

[B] R. E. Borcherds, Vertex algebras, Kac-Moody algebras, and the Monster, Proc. Natl. Acad. Sci. USA 83 (1986), 3068-3071.

[DPR] R. Dijkgraaf, V. Pasquier and P. Roche, Quasi-quantum groups related to orbifold models, Ed. by M. Carfora, M. Martellini, A Marguolis, World Scientific, 1992, 75-89.

[DVVV] R. Dijkgraaf, C. Vafa, E. Verlinde and H. Verlinde, The operator algebra of orbifold models, Comm. Math. Phys. 123 (1989), 485-526.

[D1] C. Dong, Vertex algebras associated with even lattices, J. Algebra 160 (1993), 245-265.

[D2] C. Dong, Twisted modules for vertex algebras associated with even lattices, J. Algebra 165 (1994), 91-112. 
[D3] C. Dong, Representations of the moonshine module vertex operator algebra, Contemporary Math. 175 (1994).

[DL] C. Dong, J. Lepowsky, Generalized Vertex Algebras and Relative Vertex Operators, Progress in Math. Vol. 112, Birkhäuser, Boston 1993.

[DLM] C. Dong, H. Li and G. Mason, Twisted representaions of vertex operator algebras, preprint.

[DM1] C. Dong and G. Mason, On quantum Galois theory, preprint.

[DM2] C. Dong and G. Mason, On the operator content of orbifold models, preprint.

[DMZ] C. Dong, G. Mason and Z. Zhu, Discrete series of the Virasoro algebra and the moonshine module, Proc. Symp. Pure. Math., American Math. Soc. 56 II (1994), 295-316.

[FFR] A. J. Feingold, I. B. Frenkel and J. F. X. Ries, Spinor construction of vertex operator algebras, triality and $E_{8}^{(1)}$, Contemporary Math. 121, 1991.

[FHL] I. B. Frenkel, Y.-Z. Huang and J. Lepowsky, On axiomatic approaches to vertex operator algebras and modules, Memoirs American Math. Soc. 104, 1993.

[FLM] I. B. Frenkel, J. Lepowsky and A. Meurman, Vertex Operator Algebras and the Monster, Pure and Applied Math., Vol. 134, Academic Press, 1988.

[FZ] I. Frenkel and Y. Zhu, Vertex operator algebras associated to representations of affine and Virasoro algebras, Duke Math. J. 66 (1992), 123-168.

[HL] Y.-Z. Huang and J. Lepowsky, Toward a theory of tensor products for representations of a vertex operator algebra, in: Proc. 20th Intl. Conference on Differential Geometric Methods in Theoretical Physics, New York, 1991, ed. S. Catto and A. Rocha, World Scientific, Singapore, 1992, Vol. 1, 344-354.

[J] J.C. Jantzen, Representations of Algebraic Groups, Academic Press, Orlando, 1987.

[L] H. Li, An approach to tensor product theory for representations of a vertex operator algebra, Ph.D. thesis, Rutgers University, 1994.

[Lin1] Z. Lin, Induced representations of Hopf algebras: applications to quantum groups at roots of 1, J. Algebra, 154 (1993), 152-187.

[Lin2] Z. Lin, A Mackey decomposition theorem and cohomology for the quantum groups at roots of 1, J. Algebra, 154 (1993), 152-187.

[V] D. Vogan, Representations of real reductive Lie groups, Birkhauser, Boston-Basel-Stuttgart, 1981.

[W] W. Wang, Rationality of Virasoro vertex operator algebras, Duke Math. J. IMRN, Vol. 71, No. 1 (1993), 197-211.

[Z] Y. Zhu, Vertex operator algebras, elliptic functions and modular forms, Ph.D. dissertation, Yale University, 1990.

Address: Department of Mathematics, University of California, Santa Cruz, CA 95064 dong@dong.ucsc.edu

Department of Mathematics, Kansas State University, Manhattan, KS 66506 zlin@math.ksu.edu 